\title{
Astrocytes donate mitochondria to increase glioblastoma tumorigenicity
}

Justin Lathia ( $\nabla$ lathiaj@ccf.org)

Cleveland Clinic https://orcid.org/0000-0003-3168-7290

\section{Dionysios Watson}

University Hopsitals Cleveland Medical Center

\section{Defne Bayik}

Cleveland Clinic https://orcid.org/0000-0002-4740-8869

\section{Sarah Williford}

University of Alabama

\section{Adam Lauko}

Cleveland Clinic https://orcid.org/0000-0001-5654-0784

\section{Yadi Zhou}

Cleveland Clinic

\section{Gauravi Deshpande}

Cleveland Clinic

\section{Juliana Seder}

Cleveland State University

\section{Jason Mears}

Case Western Reserve University

\section{Feixiong Cheng}

Cleveland Clinic https://orcid.org/0000-0002-1736-2847

\section{Anita Hjelmeland}

University of Alabama at Birmingham

\section{Biological Sciences - Article}

Keywords: Glioblastoma, mitochondria, tumorigenesis

Posted Date: September 30th, 2021

DOl: https://doi.org/10.21203/rs.3.rs-923533/v1

License: (9) (1) This work is licensed under a Creative Commons Attribution 4.0 International License. Read Full License 
Additional Declarations: There is NO Competing Interest. 


\section{Title: Astrocytes donate mitochondria to increase glioblastoma tumorigenicity}

Authors: Dionysios C. Watson ${ }^{1,2,3,4 \dagger}$, Defne Bayik ${ }^{1,2 \dagger}$, Sarah E. Williford ${ }^{5}$, Adam Lauko ${ }^{1,4,6}$, Yadi Zhou $^{1}$, Gauravi Deshpande ${ }^{1}$, Juliana Seder ${ }^{7}$, Jason A. Mears ${ }^{2,4}$, Feixiong Cheng ${ }^{1,2}$, Anita B.

Hjelmeland ${ }^{5}$, Justin D. Lathia ${ }^{1,2^{*}}$

\section{Affiliations:}

${ }^{1}$ Lerner Research Institute, Cleveland Clinic; 9500 Euclid Ave, Cleveland, OH 44195 USA.

${ }^{2}$ Case Comprehensive Cancer Center; 2103 Cornell Rd, Cleveland, OH 44106 USA.

${ }^{3}$ University Hospitals Cleveland Medical Center; 11100 Euclid Ave, Cleveland, OH 44106 USA.

${ }^{4}$ School of Medicine, Case Western Reserve University; 9501 Euclid Ave, Cleveland, OH 44106 USA.

${ }^{5}$ University of Alabama at Birmingham; 1918 University Blvd, Birmingham, AL 35294 USA.

${ }^{6}$ Medical Scientist Training Program, Case Western Reserve University, 9501 Euclid Ave, Cleveland, $\mathrm{OH} 44106$ USA.

${ }^{7}$ Cleveland State University; 2121 Euclid Ave, Cleveland, OH 44115 USA.

*Corresponding author. Email: lathiaj@ccf.org

$\dagger$ These authors contributed equally to this work. 


\section{SUMMARY PARAGRAPH:}

Glioblastoma (GBM), the most common primary brain cancer in adults ${ }^{1}$, is characterized by numerous cell-intrinsic/extrinsic interactions that drive tumorigenesis. In addition to cellsurface and secreted protein/extracellular vesicle interactions ${ }^{2,3}$, treatment resistance of GBM is augmented by the formation of cytoplasmic interconnections and junctions among tumor cells ${ }^{4}$. These cytoplasmic bridges among tumor cells enable exchange of cellular metabolites as well as mitochondria ${ }^{4}$, which can play a key role in metabolic function and cellular programming in $\mathrm{GBM}^{5,6}$. However, the contribution of the tumor microenvironment to mitochondrial transfer, as well as the downstream impact of mitochondrial transfer on GBM remains poorly characterized. Here we identified horizontal mitochondrial transfer from the tumor microenvironment as a mechanism that enhances tumorigenesis in glioblastoma. We found that this transfer occurs primarily from brain-resident cells, including astrocytes, and can be appreciated in vitro and in vivo through intercellular connections between GBM cells and non-malignant host cells. The acquisition of astrocyte mitochondria drives an overall enhancement of mitochondrial membrane potential and metabolic capacity, while increasing glioblastoma cell self-renewal and tumor-initiating capacity. Collectively, our findings demonstrate that astrocyte mitochondrial transfer augments the tumorigenic capacity of glioblastoma cells and reveals a previously unknown cell-cell communication mechanism that drives tumor growth. We anticipate our findings will open new research directions to decipher the molecular events linking mitochondria acquisition from non-malignant cells to increased tumorigenicity of recipient GBM cells. This line of research will lead to new therapeutic opportunities targeting this understudied phenomenon and its sequelae in GBM.

\section{Main Text}

While dynamic microenvironmental interactions drive tumor growth and therapeutic resistance in $\mathrm{GBM}^{2,3}$, the underlying direct cell-cell communication mechanisms remain poorly understood. Recent studies established that mitochondrial transfer in mouse models following ischemic stroke was important for neuronal recovery ${ }^{7}$. Given the crucial role of brain microenvironmental interactions in driving GBM pathobiology ${ }^{8}$, we hypothesized that acquisition of mitochondria from the tumor microenvironment results in metabolic changes and augmented tumorigenicity in GBM cells.

To assess whether non-malignant host cells also transfer mitochondria to GBM in vivo, we orthotopically implanted GFP-expressing syngeneic mouse GBM models (SB28 and GL261) into transgenic C57BL/6 mice expressing a mitochondria-localized mKate2 fluorophore by fusion to the localization peptide of cytochrome c oxidase 8 (mito::mKate 2 mice $^{9}$ ), Fig. 1A). Confocal microscopy of GBM tumors from mito::mKate 2 mice revealed mKate $2^{+}$puncta within $15-50 \%$ of $\mathrm{GFP}^{+}$GBM cells (Fig. 1B-C, fig. S1), demonstrating that host cell mitochondria were being transferred to GBM cells in vivo. By co-staining tissue sections with wheat germ agglutinin to highlight glycoprotein-rich structures ${ }^{10}$, we observed that transferred mitochondria frequently localized at host-tumor interfaces and at the termini of intercellular connections between $\mathrm{GFP}^{+} \mathrm{GBM}$ cells and $\mathrm{GFP}^{-}$host cells containing $\mathrm{mKate} 2^{+}$mitochondria (Fig. 1B, fig. S2-3). Thus, cytoplasmic interconnections previously reported to exist among GBM cells ${ }^{4}$ likely also play a role in organelle transfer between GBM and microenvironment cells.

Having observed mitochondrial transfer from the tumor microenvironment to GBM cells in mouse models, we proceeded to interrogate the identity of the host mitochondrial donor cells. 
GBM tumors in mouse models and patients are known to have significant infiltration by both brain-resident glia and peripheral immune cells that transmigrate into the microenvironment ${ }^{8}$. We established orthotopic GBM tumors in wildtype C57BL/6 mice that had first received lethal irradiation with subsequent bone marrow reconstitution from mito::mKate2 mice (mito::mKate2 $\rightarrow$ WT) to restrict mKate2 expression to immune cells (Fig. 1A). Analysis of single-cell suspensions by flow cytometry indicated negligible host mitochondrial transfer to $\mathrm{GFP}^{+}$GBM cells in mito::mKate $\rightarrow$ WT mice, while $20-60 \%$ of $\mathrm{GFP}^{+}$GBM cells detected were $m$ Kate $2^{+}$in mito::mKate2 mice (Fig. 1D-E). Taken together, these data suggest that brain-resident cells and not tumor-infiltrating immune cells were the major donors of mitochondria to GBM cells in vivo.

To further elucidate the identity of the predominant mitochondrial donor cell populations, we co-cultured prevalent tumor-infiltrating cell types with $\mathrm{GFP}^{+} \mathrm{GBM}$ cells at a 1:2 donor-torecipient ratio. After 2 hours, we assessed the percentage of mKate 2 positivity as a marker of mitochondrial transfer (Fig. 2A). Consistent with our in vivo observations, we found that brainresident glia (astrocytes and microglia) donated significantly more mitochondria on a per-cell basis compared to bone marrow-derived macrophages (Fig. 2B, fig. S4A). While further polarization of macrophages to an M2- or M1-like phenotype potentially favored increased mitochondrial transfer, the degree of transfer was on average 5-10-fold less than that observed with the brain-resident glia in vitro (fig. S4B). In addition, culturing GBM cells with donor cell-conditioned medium did not result in mKate2 signal transfer to tumor cells, further suggesting that the mitochondrial transfer is primarily contact dependent (Fig. 2C, fig. S4C). Live confocal imaging of astrocyte/SB28 cell co-cultures confirmed the occurrence of mitochondrial transfer at donor-recipient contact sites (Fig. 2D, Movie S1), consistent with our in vivo observation that host-tumor intercellular connections contained host mitochondria (fig. S2-3). Taken together, these results indicate that physical interaction of tumor cells with donor cells is required for effective mitochondrial transfer.

To interrogate the functional significance of mitochondria acquisition, we sorted $\mathrm{mKate} 2^{+}$ (with exogenous mitochondria) and mKate2- (without exogenous mitochondria) GBM cells from mito::mKate2 astrocyte co-cultures after 2 days (Fig. 3A). Unsupervised clustering of RNA-seq analysis revealed that tumor cells had a distinct gene expression profile compared to astrocytes, confirming that sorted $\mathrm{mKate} 2^{+} \mathrm{SB} 28$ cells were not significantly contaminated by astrocytes from the co-culture (fig. S5A-C). Additionally, there were a limited number of differentially expressed genes between $\mathrm{mKate}^{+}$and $\mathrm{mKate} 2^{-}$cells, indicating that mitochondrial transfer in vitro does not induce broad transcriptional changes (fig. S5D, Table S1). However, not surprisingly, genes that were upregulated $>1.5$-fold in $m K a t e 2^{+}$samples were enriched within pathways related to mitochondrial biology, in particular electron transport and mitochondrial organization (Fig. 3B, fig. S6). In line with this finding, mKate $2^{+}$ GBM cells exhibited increased mitochondrial membrane potential and mass compared to their mKate2- counterparts, as measured by MitoStatus and MitoTracker Deep Red staining (Fig. 3C, fig. S7A, fig. S7B). When normalized to total mitochondrial content, mitochondrial potential remained higher in $\mathrm{mKate} 2^{+}$cells (fig. S7A, fig. S7C).

To further test the metabolic consequences of acquiring astrocyte mitochondria, we assessed metabolic activity via Seahorse assays, as well as expression of key metabolic proteins by adapting a previously reported metabolic flow cytometry panel ${ }^{11}$. Metabolic profiling of sorted cells revealed that both mKate $2^{+}$and $m K a t e 2^{-}$GBM cells had a lower oxygen consumptionto-extracellular acidification ratio (OCR/ECAR) than matched astrocytes, suggesting that they 
rely more heavily on glycolytic metabolism in the glucose-rich assay environment, in line with the observed high and invariable GLUT1 expression among these GBM cell subsets (fig. S8A, $\mathbf{8 E}, 9)$. In contrast, despite similar rates of mitochondrial respiration in the presence of excess glucose, mKate $2^{+}$cells had higher levels of ATP5A (mitochondrial ATP synthase), SLC20A1 (cellular phosphate import) and ACC1 (fatty acid synthesis), pointing to potential differences in the cellular energetics of mitochondria recipients. Increased ATP5A protein expression was also observed in mKate $2^{+} \mathrm{GBM}$ cells isolated from intracranial tumors implanted in mito::mKate2 mice (data not shown).

To assess whether mitochondrial acquisition impacts the tumorigenic potential of GBM cells, we utilized an in vitro limiting dilution assay and found that mKate ${ }^{+} \mathrm{SB} 28$ cells had a higher self-renewal capacity compared to matching cells without exogenous mitochondria, with an estimated stem cell frequency that was $\sim 2.5$-fold higher (Fig. 4A, fig. S10A). We further performed an in vivo limiting dilution assay by intracranial implantation of decreasing numbers of $\mathrm{mKate} 2^{+}$and mKate2- SB28 cells. In three independent experiments, we consistently observed that cohorts receiving $\mathrm{mKate} 2^{+} \mathrm{GBM}$ cells displayed increased penetrance and/or earlier symptomatic disease (fig. S10B), corresponding to an estimated 3-fold higher frequency of tumor-initiating cells (Fig. 4B). Finally, $\sim 10-20 \%$ of patient-derived xenograft (PDX) cells also demonstrated the ability to acquire mitochondria from human astrocytes in co-culture, as assessed by flow cytometry and ImageStream in two separate lines (Fig. 4C, fig. S11). Taken together, these data demonstrate increased tumorigenicity of cells that received astrocytederived mitochondria and suggest that this mode of intercellular communication could play a role in human GBM tumors by altering cellular energetics (Fig. 4D).

Organelle transfer is an increasingly recognized biological process in models of $\mathrm{GBM}^{12-14}$ and other cancers ${ }^{15-17}$. However, there is a lack of understanding about its in vivo relevance, specific donor-recipient interactions and downstream effects. We found that in the context of GBM, this is a frequent in vivo event, involving primarily brain-resident glial cell donors, and is associated with recipient GBM cells acquiring a more tumorigenic phenotype. Mitochondrial transfer led to changes in key metabolic proteins and conferred an advantage to GBM cells within the brain microenvironment. Additional studies into the mechanisms of transfer, as well as into the molecular changes in the recipient cells, are crucial to expand our understanding of this observation and lead to the identification of a new class of therapeutic targets. This line of research could also have broad applicability to tumors outside the central nervous system.

Supplementary Information is available for this paper.

\section{References}

1 Ostrom, Q. T. et al. CBTRUS Statistical Report: Primary Brain and Other Central Nervous System Tumors Diagnosed in the United States in 2013-2017. Neuro Oncol 22, iv1-iv96, doi:10.1093/neuonc/noaa200 (2020).

2 Yin, J. et al. Exosomal transfer of miR-1238 contributes to temozolomide-resistance in glioblastoma. EBioMedicine 42, 238-251, doi:10.1016/j.ebiom.2019.03.016 (2019).

3 Knudsen, A. M., Rudkjobing, S. J., Sorensen, M. D., Dahlrot, R. H. \& Kristensen, B. W. Expression and Prognostic Value of the Immune Checkpoints Galectin-9 and PD-L1 in Glioblastomas. J Neuropathol Exp Neurol 80, 541-551, doi:10.1093/jnen/nlab041 (2021).

4 Osswald, M. et al. Brain tumour cells interconnect to a functional and resistant network. Nature 528, 93-98, doi:10.1038/nature16071 (2015). 
5 Park, H. K. et al. Interplay between TRAP1 and Sirtuin-3 Modulates Mitochondrial Respiration and Oxidative Stress to Maintain Stemness of Glioma Stem Cells. Cancer Res 79, 1369-1382, doi:10.1158/0008-5472.CAN-18-2558 (2019).

6 Khacho, M. et al. Mitochondrial Dynamics Impacts Stem Cell Identity and Fate Decisions by Regulating a Nuclear Transcriptional Program. Cell Stem Cell 19, 232-247, doi:10.1016/j.stem.2016.04.015 (2016).

7 Hayakawa, K. et al. Transfer of mitochondria from astrocytes to neurons after stroke. Nature 535, 551-555, doi:10.1038/nature18928 (2016).

8 Broekman, M. L. et al. Multidimensional communication in the microenvirons of glioblastoma. Nat Rev Neurol 14, 482-495, doi:10.1038/s41582-018-0025-8 (2018).

9 Barrasso, A. P., Tong, X. \& Poche, R. A. The mito::mKate2 mouse: A far-red fluorescent reporter mouse line for tracking mitochondrial dynamics in vivo. Genesis 56, doi:10.1002/dvg.23087 (2018).

10 Tsai, I. J., Su, E. C., Tsai, I. L. \& Lin, C. Y. Clinical Assay for the Early Detection of Colorectal Cancer Using Mass Spectrometric Wheat Germ Agglutinin Multiple Reaction Monitoring. Cancers (Basel) 13, doi:10.3390/cancers13092190 (2021).

11 Ahl, P. J. et al. Met-Flow, a strategy for single-cell metabolic analysis highlights dynamic changes in immune subpopulations. Commun Biol 3, 305, doi:10.1038/s42003-020-10279 (2020).

12 Civita, P., D, M. L. \& Pilkington, G. J. Pre-Clinical Drug Testing in 2D and 3D Human In Vitro Models of Glioblastoma Incorporating Non-Neoplastic Astrocytes: Tunneling Nano Tubules and Mitochondrial Transfer Modulates Cell Behavior and Therapeutic Respons. Int J Mol Sci 20, doi:10.3390/ijms20236017 (2019).

13 Pinto, G. et al. Patient-derived glioblastoma stem cells transfer mitochondria through tunneling nanotubes in tumor organoids. Biochem J 478, 21-39, doi:10.1042/BCJ20200710 (2021).

14 Valdebenito, S. et al. Tunneling nanotubes, TNT, communicate glioblastoma with surrounding non-tumor astrocytes to adapt them to hypoxic and metabolic tumor conditions. Sci Rep 11, 14556, doi:10.1038/s41598-021-93775-8 (2021).

Dong, L. F. et al. Horizontal transfer of whole mitochondria restores tumorigenic potential in mitochondrial DNA-deficient cancer cells. Elife 6, doi:10.7554/eLife.22187 (2017).

16 Pasquier, J. et al. Preferential transfer of mitochondria from endothelial to cancer cells through tunneling nanotubes modulates chemoresistance. J Transl Med 11, 94, doi:10.1186/1479-5876-11-94 (2013).

17 Marlein, C. R. et al. CD38-Driven Mitochondrial Trafficking Promotes Bioenergetic Plasticity in Multiple Myeloma. Cancer Res 79, 2285-2297, doi:10.1158/00085472.CAN-18-0773 (2019). 
A

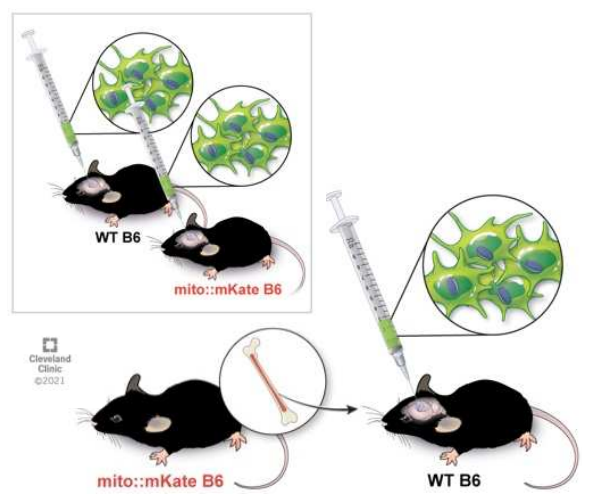

B
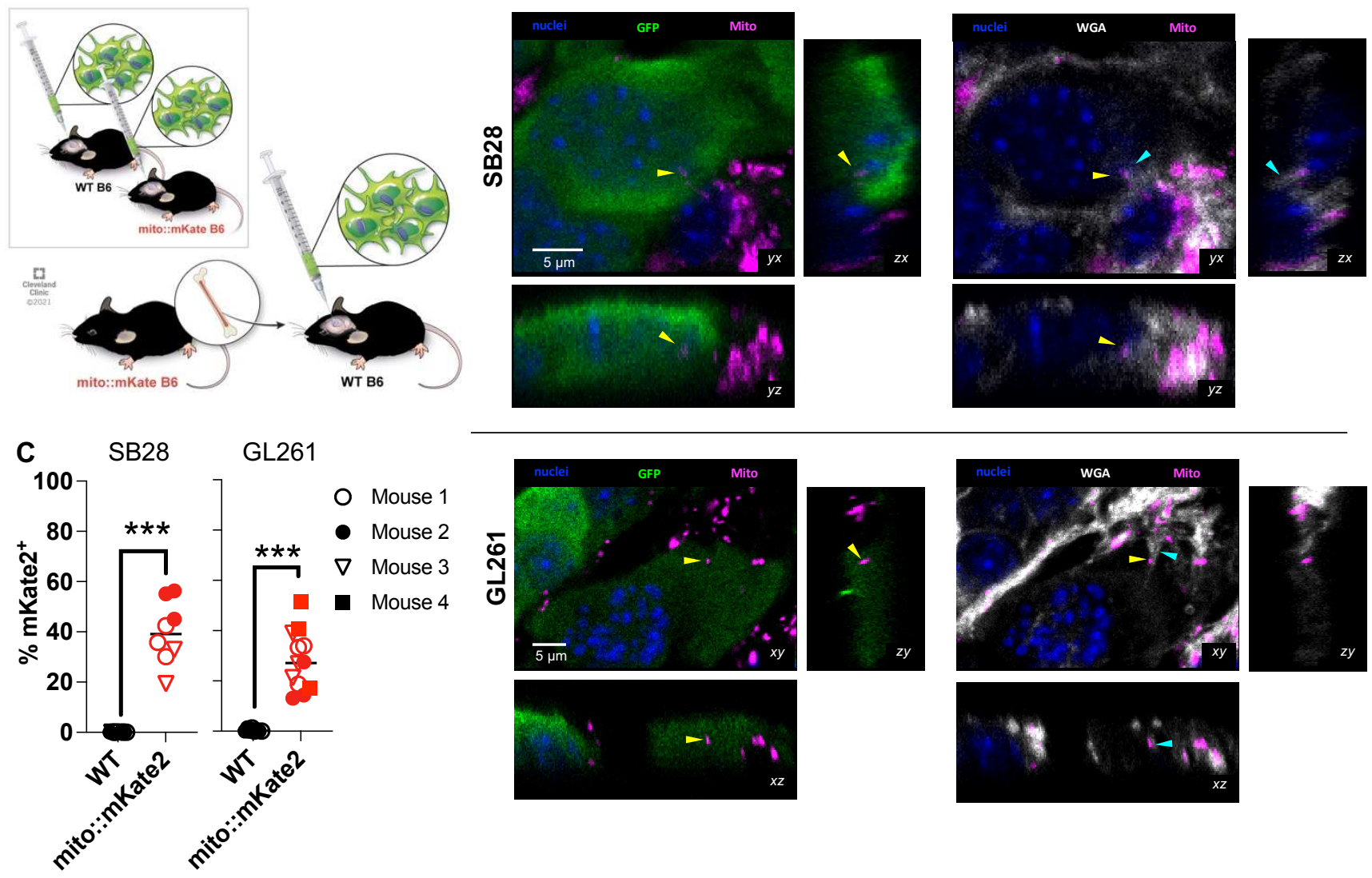

mito::mKate2 $\rightarrow$ WT

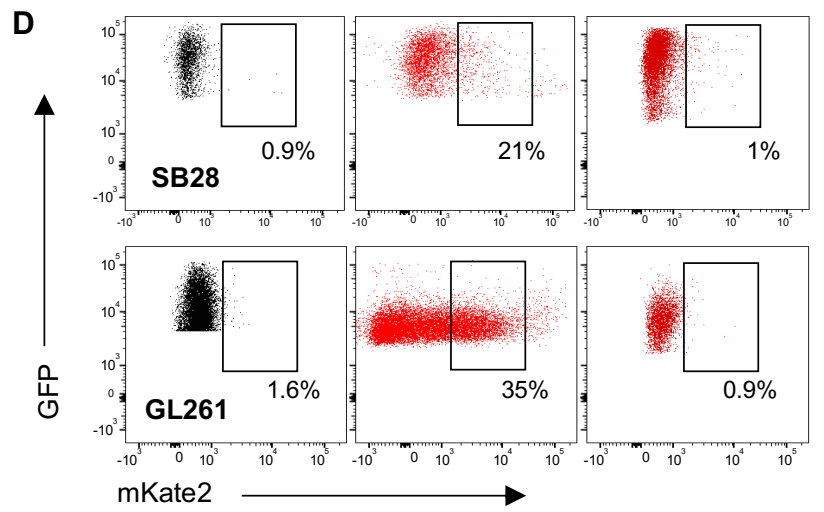

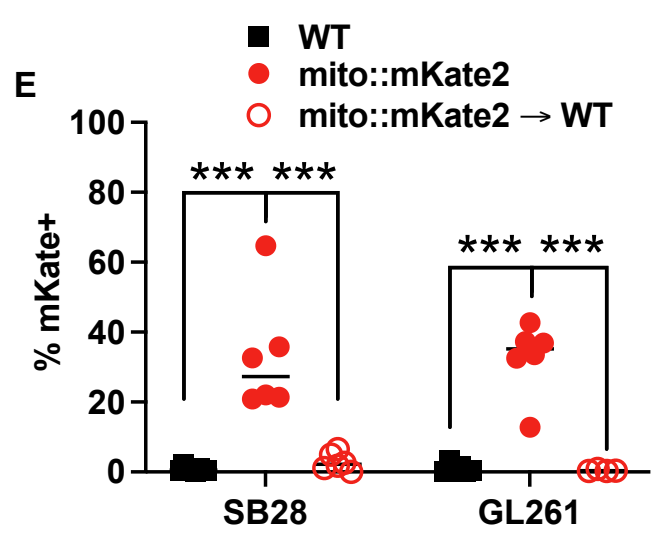

Fig. 1. GBM cells obtain host mitochondria from the tumor microenvironment. (A) GFPexpressing SB28 and GL261 cells were implanted intracranially into wild-type (WT), mKate2::mito (mKate2) or WT mice with mito::mkate2 bone marrow (mito::mKate2 $\rightarrow$ WT). The assays below were performed at endpoint. (B) Single focal planes (xy) and z-stack orthogonal reconstructions $(\mathrm{xz}, \mathrm{yz})$ at areas of tumor/host cell interface. Yellow arrowheads point to host mKate + mitochondria within recipient tumor cells. Cyan arrowheads point to wheat germ agglutinin (WGA)-labelled tether-like structures. (C) 3D confocal imaging segmentation-based estimation of $\mathrm{mKate} 2^{+} \mathrm{GFP}^{+} \mathrm{GBM}$ cell frequency from 2-3 visual fields from $\mathrm{n}=2-4$ mice/group. $* * * \mathrm{p}<0.001$, t-test. (D) Representative dot plots and (E) quantification of relative frequency of 
mito::mKate ${ }^{+} \mathrm{GFP}^{+} \mathrm{GBM}$ cells by flow cytometry; $\mathrm{n}=4-8$ per group. ${ }^{* * *} \mathrm{p}<0.001$, 2-way ANOVA. 
A

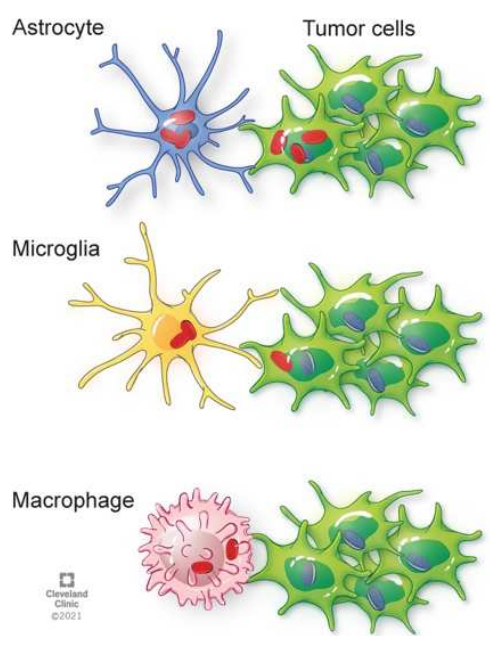

C

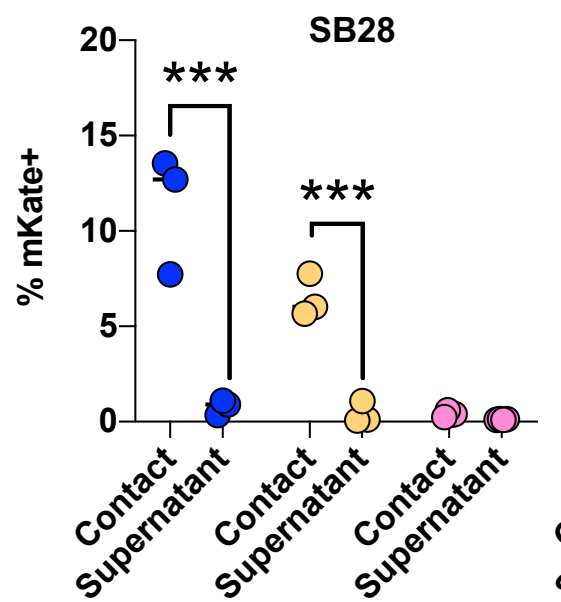

B

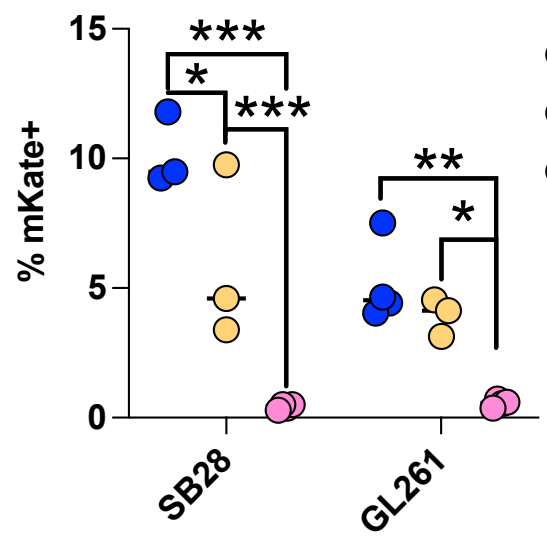

- Astrocytes

O Microglia

O M0 macrophages

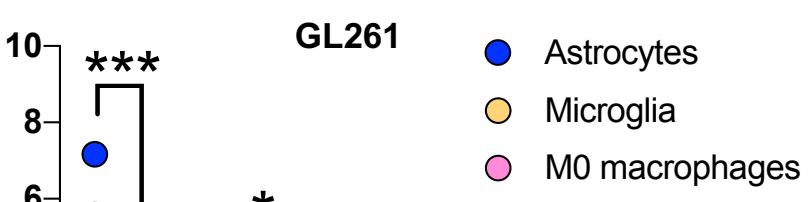

D Pre-transfer contact points

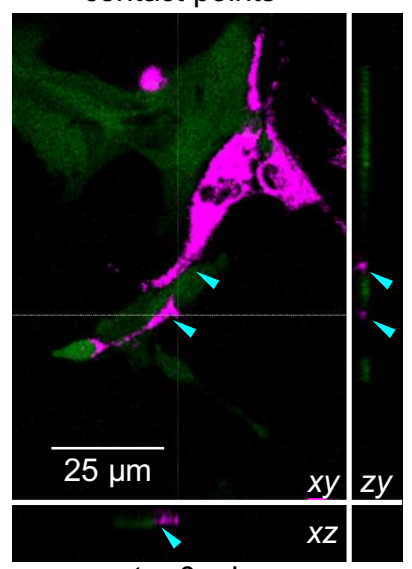

$\mathrm{t}=0 \mathrm{~min}$
mito::mKate2 transfer to dividing GBM cell

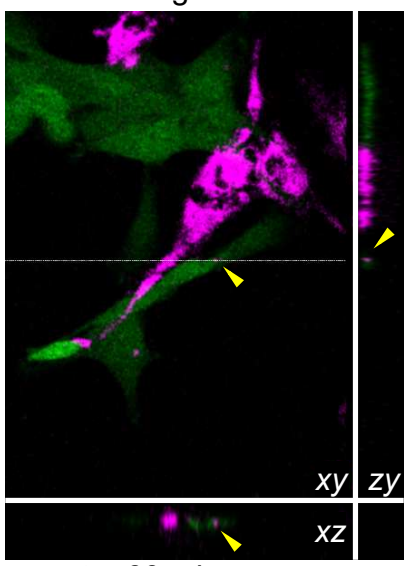

$\mathrm{t}=60 \mathrm{~min}$
mito::mKate2 retention in GBM cell

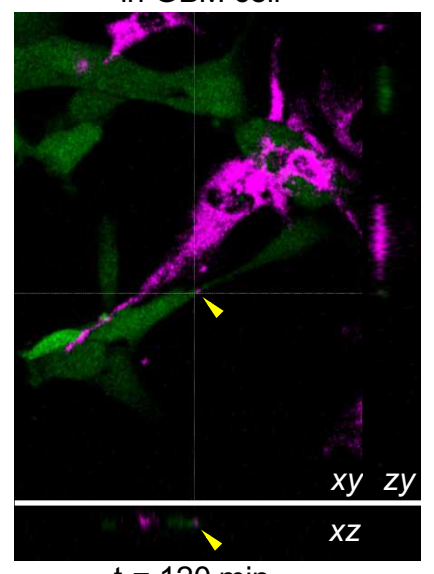

$\mathrm{t}=120 \mathrm{~min}$
mito::mKate2 movement among GBM cells

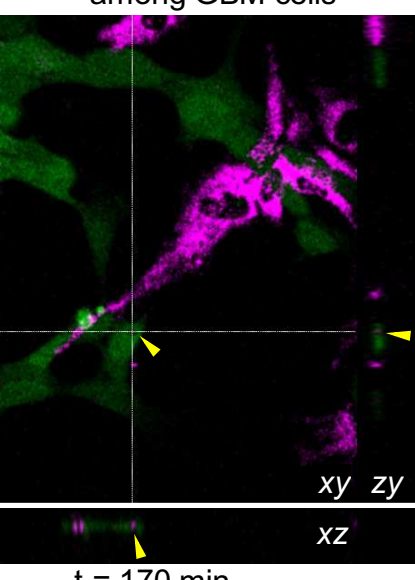

Fig. 2. Astrocytes horizontally transfer mitochondria to GBM cells in a contact-dependent manner. (A) GBM cells were co-cultured with astrocytes, microglia or macrophages from mito::mKate 2 mice for 2 hours, and mitochondrial transfer was analyzed by flow cytometry. (B) Relative frequency of $\mathrm{mKate} 2^{+} \mathrm{GFP}^{+} \mathrm{GBM}$ cells. (C) Relative frequency of contact-dependent (contact) versus contact-independent (supernatant) mitochondrial transfer. $n=3-4$ biological 
replicates for B, C. $* \mathrm{p}<0.05, * * \mathrm{p}<0.01, * * * \mathrm{p}<0.001,2$-way ANOVA. (D) Confocal time-lapse images demonstrating real-time acquisition of astrocyte mitochondria (magenta) by SB28 cells (green). 
A

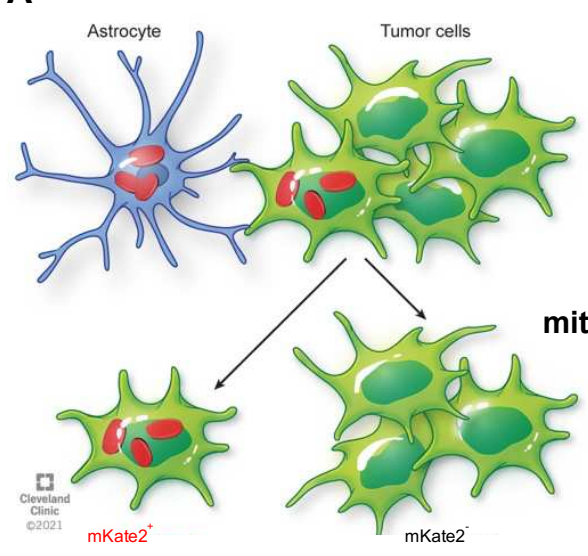

C

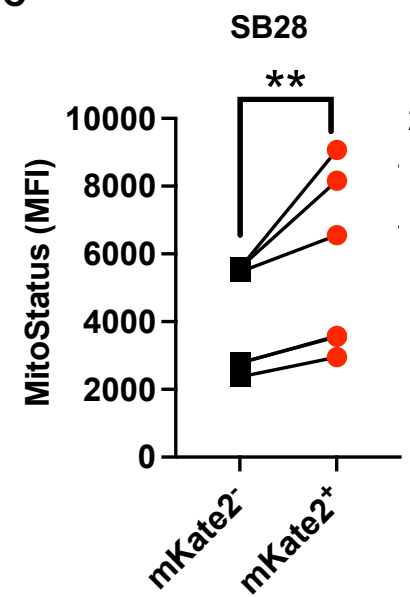

B

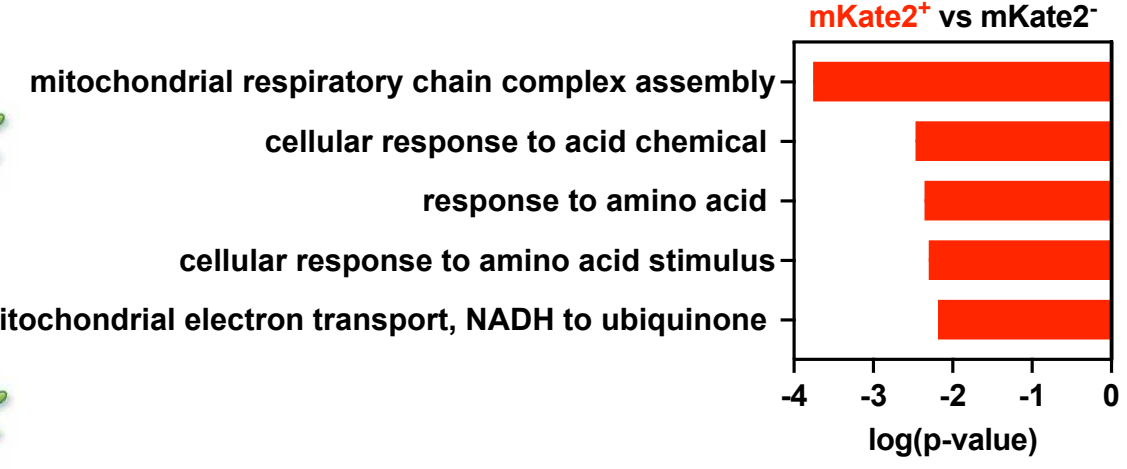

D
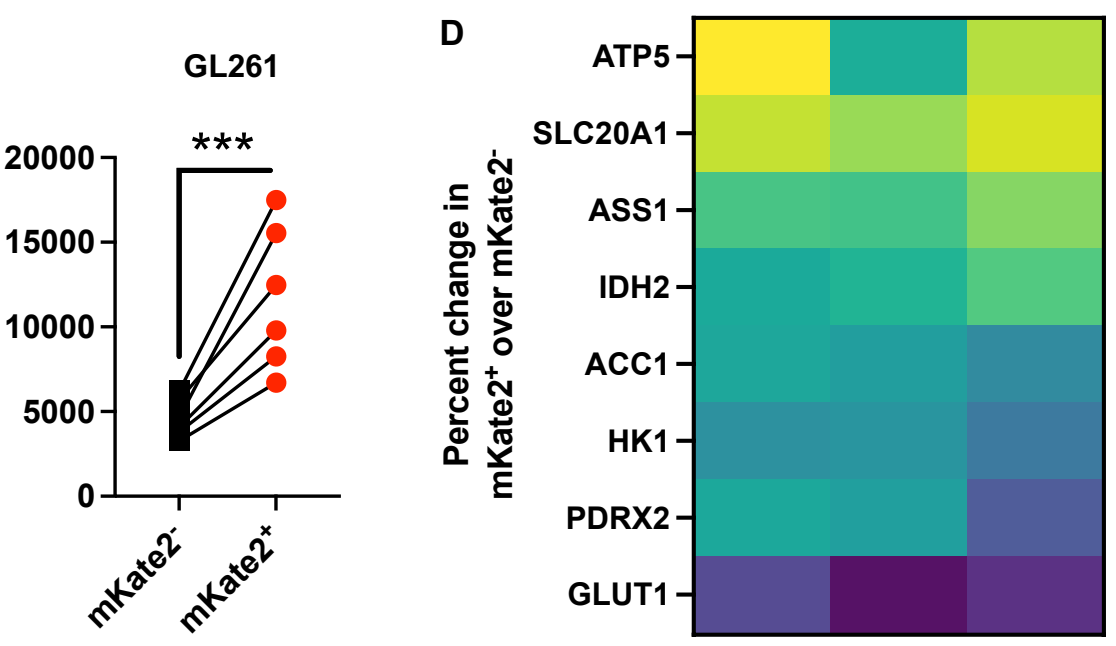

20

15

Fig. 3. Tumor cells receiving donor mitochondria have altered cellular metabolism. (A) GBM cells were co-cultured with astrocytes from mito::mKate 2 mice for $48 \mathrm{~h}$. mKate $2^{+}$and mKate2SB28 cells were sorted by flow cytometry for downstream assays. (B) The top 5 pathways enriched in $m$ Kate $2^{+}$versus mKate $2^{-}$SB28 cells based on genes upregulated $>1.5$ fold with $p<0.05$. (C) Geometric mean fluorescence intensity (MFI) of mitochondrial membrane potential dye MitoStatus in mKate $2^{+}$versus mKate $2^{-}$GBM cells co-cultured with astrocytes for $48 \mathrm{~h}$; $\mathrm{n}=5-6$ per group. $* * \mathrm{p}<0.01, * * * \mathrm{p}<0.001$, t-test. (D) Percent change in MFI of key metabolic pathway proteins in $\mathrm{mKate} 2^{+}$versus $\mathrm{mKate} 2^{-} \mathrm{SB} 28$ cells co-cultured with astrocytes for $24 \mathrm{~h}$, as measured by flow cytometry. 

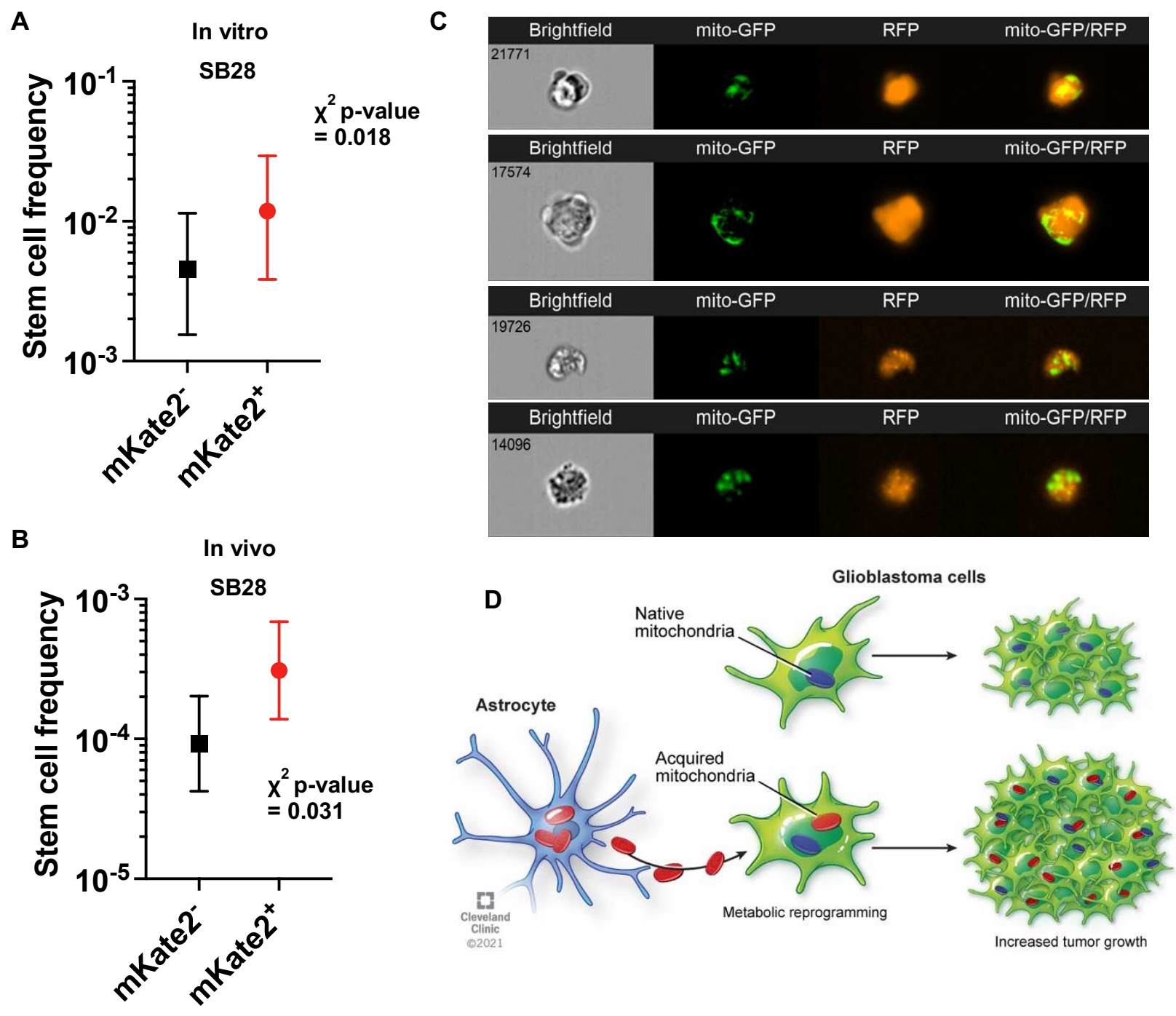

Fig. 4. Mitochondrial transfer enhances tumor-initiating capacity. (A) Sorted tumor cells were cultured at decreasing cell densities, and the number of wells containing spheres was counted 14 days later. Representative data from $n=3$ experiments shown as mean $\pm 95 \%$ confidence interval. (B) Decreasing concentrations of $m K$ ate $2^{+}$and mKate $2^{-} \mathrm{SB} 28$ cells were implanted into $\mathrm{n}=4-5$ mice. The number of animals that developed tumors within 60 days was used to calculate tumorforming cell frequency. (C) ImageStream was used to visualize transfer of GFP-labelled mitochondria from human astrocytes to RFP-expressing D456 patient-derived xenografts. (D) GBM cells acquiring exogenous mitochondria undergo metabolic reprogramming and have enhanced tumorigenicity. 


\section{Methods}

Mouse tumor cell maintenance and transduction

SB28 cells were gifted by Dr. Hideho Okada (University of California, San Francisco). GL261 cells were obtained from the Developmental Therapeutics Program, National Cancer Institute. All cell lines were treated with 1:100 MycoRemoval Agent (MP Biomedicals) upon thawing and routinely tested for Mycoplasma spp. (Lonza). Cells were maintained in RPMI 1640 (Media Preparation Core, Cleveland Clinic) supplemented with 10\% FBS (Thermo Fisher Scientific) and 1\% penicillin/streptomycin (1\% Pen/Strep, Media Preparation Core). For the generation of GFP-expressing GL261 cells, parental GL261 cells were transduced with pReceiverLv207 (Genecopoeia) and were selected with $300 \mu \mathrm{g} / \mathrm{ml}$ hygromycin B (Invitrogen). GFP expression was confirmed by flow cytometry.

Mice

All animal experiments were approved by the Institutional Animal Care and Use Committee of Cleveland Clinic and performed in accordance with established guidelines. $\operatorname{Tg}(\mathrm{CAG}-$ mKate2)1Poche/J (mito::mKate2, stock \#032188) mice were purchased from The Jackson Laboratory and were housed in the Cleveland Clinic Biological Research Unit. Both sexes of mito::mKate2 mice were intracranially injected at 4-8 weeks old with 10,000-20,000 SB28 or 100,000 GL261-GFP cells in $5 \mu$ RPMI null media into the left cerebral hemisphere $2 \mathrm{~mm}$ caudal to the coronal suture, $3 \mathrm{~mm}$ lateral to the sagittal suture at a $90^{\circ}$ angle with the murine skull to a depth of $2.5 \mathrm{~mm}$, using a stereotaxis apparatus (Kopf). Age- and sex-matched wildtype littermates were used as controls. Mice were monitored daily for neurological symptoms, lethargy and hunched posture that would qualify as signs of tumor burden.

25 Bone marrow transplantation

Four-week-old male mice were treated with 11 Gy radiation in two fractions 3-4 hours apart. Reconstitution was achieved by retro-orbital injection of $2 \times 10^{6}$ bone marrow cells from mito::mKate2 mice. Drinking water was supplemented with Sulfatrim (trimethoprimsulfamethoxazole; Pharmaceutical Associates, Inc.) during the first 10 days, and mice were

30 monitored for an additional 6 weeks for weight loss and symptoms of infection before tumor inoculation. Survival analysis was performed as described above.

\section{Assessment of mitochondrial trafficking in vivo by confocal microscopy}

At experiment endpoint, mice were perfused with 4\% paraformaldehyde in PBS (4\% PFA, Fisher Chemical) using the Perfusion One system (Leica). Brains were dissected and stored for 48 $\mathrm{h}$ in $20 \mathrm{ml} 4 \%$ PFA at $4^{\circ} \mathrm{C}$, transferred to $20 \mathrm{~mL}$ PBS for $48 \mathrm{~h}$ at $4^{\circ} \mathrm{C}$, and finally transferred to 20 $\mathrm{mL} 30 \%$ sucrose (Fisher Chemical) in PBS at $4^{\circ} \mathrm{C}$ until density equilibration (typically $\sim 48 \mathrm{~h}$ ). Samples were embedded in OCT Compound (Tissue-Tek), flash frozen and stored at $-80^{\circ} \mathrm{C}$. Ten micron sections were prepared using a cryostat (Leica Biosystems), immediately mounted on charged glass slides (Superfrost Plus, Fisherbrand) and stored at $-20^{\circ} \mathrm{C}$ until staining. For staining, selected slides were allowed to warm to room temperature, washed twice with $0.1 \%$ Triton-X 100 (Sigma) in PBS (PBS-T) for $10 \mathrm{~min}$, and permeabilized overnight at $4{ }^{\circ} \mathrm{C}$ with $5 \%$ donkey serum (Jackson ImmunoResearch), 0.3\% Triton-X 100, $1 \mathrm{mg} / \mathrm{mL}$ bovine serum albumin (molecular biology grade, Sigma) in PBS. Sections were then stained with chicken anti-GFP antibody (1:1000 in permeabilization buffer; clone AB_2307313, AvesLabs) overnight at $4^{\circ} \mathrm{C}$. Sections were then washed 3 times with PBS-T for 10 min and stained with donkey anti-chicken Alexa fluor (AF) 
488-conjugated secondary antibody (1:500 in permeabilization buffer, AB_2340375, Jackson ImmunoResearch) overnight at $4^{\circ} \mathrm{C}$. Sections were then washed 3 times with PBS-T for $10 \mathrm{~min}$, then exchanged to HBSS (without phenol red) with $\mathrm{CaCl}_{2}, \mathrm{MgCl}_{2}, \mathrm{MgSO}_{4}$ and $0.1 \%$ Triton-X 100 (HBSS-T) by washing 3 times for $10 \mathrm{~min}$. Sections were immediately stained with wheat germ agglutinin conjugated to AF680 (4 $\mu \mathrm{g} / \mathrm{mL}$ in HBSS-T; ThermoFisher) for 1 hour at room temperature and washed 3 times with HBSS-T for $10 \mathrm{~min}$. Nuclear staining was performed with Hoechst $33342(3.3 \mu \mathrm{g} / \mathrm{mL}$ in PBS-T). Finally, stained sections were mounted and coverslipped with Vectashield Vibrance and no. 1.5 glass coverslips (Fisherbrand). Mounting medium was given at least 24 hours to cure prior to imaging.

Confocal microscopy of stained tissue sections was performed using a Leica SP8. Fullthickness Z-stacks were obtained at 3-4 optical fields at sites of tumor-microenvironment interface of each sample using a $63 x$ lens. Still image processing and z-reconstructions were completed using LasX software (version 3.3, Leica). Image analysis for estimation of mito::mKate2 transfer to GBM cells in vivo was performed using Velocity software (version 6.3, PerkinElmer). The 3D segmentation algorithms (see Supplementary Text) were set to minimize: (a) detection of mKate2-channel noise (using wildtype tissue sections as a negative control) and (b) identification of GFP+ cells not morphologically compatible with tumor cells, likely the result of GFP phagocytosis in the tumor microenvironment (using a relevant size cutoff).

Measurement of mitochondrial trafficking in vivo by flow cytometry

At experiment endpoint, mice were euthanized, and resected tumors or the contralateral hemisphere were digested with $1 \mathrm{mg} / \mathrm{mL}$ collagenase IV (StemCell Technologies) and $1 \mathrm{mg} / \mathrm{mL}$ DNAse I (Roche) for 15 minutes at $37^{\circ} \mathrm{C}$. Samples were strained trough a $100 \mu \mathrm{m}$ strainer (Fisherbrand) and washed with PBS. Cells were stained with LIVE/DEAD ${ }^{\mathrm{TM}}$ Fixable Blue Dead Cell Stain Kit (Thermo Fisher Scientific) for 10 minutes on ice, treated with 1:50 diluted FcR blocking reagent (Miltenyi Biotec) for 15 minutes on ice and stained with 1:100 APC-conjugated anti-CD11b antibody (Biolegend, clone M1/70) for 20 minutes to exclude phagocytic cells. Samples were fixed overnight with eBioscience ${ }^{\mathrm{TM}}$ FoxP3 Transcription Factor Fixation Kit (Thermo Fisher Scientific) and analyzed with a BD LSRII Fortessa (BD Biosciences) in PBS.

Generation of mouse astrocytes and microglia and in vitro mitochondria transfer assay

Brain-resident glial cell cultures were obtained as previously described ${ }^{1}$. Briefly, mito::mKate 2 mice at post-natal day 0-3 were euthanized, and mito::mKate2 signal was confirmed by an EVOS Cell Imaging System (Thermo Fisher Scientific). Brain subventricular zones (SVZs) were digested in $4 \mathrm{~mL}$ Accutase Cell Dissociation Reagents (Biolegend) for $5 \mathrm{~min}$ and washed with $10 \mathrm{~mL}$ neural stem cell (NSC) media: DMEM:F12 (Media Preparation Core), 1\% Pen/Strep, 5\% FBS, 2\% N2 supplement (Thermo Fisher Scientific), $20 \mathrm{ng} / \mathrm{mL}$ EGF and $20 \mathrm{ng} / \mathrm{mL}$ FGF-2 (R\&D Systems). Cells were strained through a $70 \mu \mathrm{m}$ strainer (Fisherbrand) and cultured into T25 flasks (Corning) in $10 \mathrm{~mL}$ NSC media. At confluency, cells were split into two and transferred into T75 flasks (USA Scientific). One flask was kept in NSC media to support astrocyte growth and was split bi-weekly and not used beyond passage 10. The media of the second flask was replaced with microglia polarization media (MPM): DMEM:F12, 1\% Pen/Strep, 10\% FBS, 20 $\mathrm{ng} / \mathrm{mL}$ GM-CSF (Biolegend) when the cells reached confluency. Seven days later, microglia growing on the underlying feeder cell layer were shaken off for 1 hour.

A total of 20,000-40,000 astrocytes and microglia were separately cultured in a 96-well flat-bottom plate (Thermo Fisher Scientific) in NSC medium or MPM. Forty-eight hours later, supernatants were collected and centrifuged at $400 \mathrm{~g}$ for 5 minutes to remove residual cells. Tumor 
cells were added at a recipient:donor ratio of 2:1. Samples were incubated for 2 hours and treated with Accutase to generate single-cell suspensions. Cells were transferred to 96-well U-bottom plates (Thermo Fisher Scientific) to stain with Live/Dead dye. Exogenous mitochondria uptake of $\mathrm{GFP}^{+}$tumor cells was assessed with a BD LSRII Fortessa.

Generation of mouse macrophages and in vitro mitochondria uptake assay

Bone marrow from the femur and tibia of 4-8-week-old male and female mito::mKate2 mice was flushed with PBS using a $27 \mathrm{G}$ needle. A total of 80,000 cells was cultured in 24-well plates (Corning) and treated with $50 \mathrm{ng} / \mathrm{ml}$ recombinant mouse M-CSF (Biolegend) in IMDM (Media Preparation Core) supplemented with 1\% Pen/Strep and 20\% FBS for 6 days. IFN $\gamma$ or IL$4(50 \mathrm{ng} / \mathrm{ml}$; Biolegend) was added for 48 hours to further induce polarization of macrophages to M1- or M2-like macrophages. Supernatants were collected and centrifuged at $400 \mathrm{~g}$ for 5 minutes to remove residual cells. Tumor cells were added at 2-fold abundance in technical duplicates for a 2-hour incubation. Samples were incubated with Accutase for 5 minutes and transferred into 96well U-bottom plates for staining with the viability dye and anti-CD11b antibody as described above. Exogenous mitochondria uptake was analyzed from $\mathrm{GFP}^{+}$tumor cells using a BD LSRII Fortessa.

\section{Confocal microscopy time-lapse imaging}

SB28 cells $(40,000)$ were co-cultured with 80,000 mito::mKate2 astrocytes in a glassbottom $35 \mathrm{~mm}$ dish (Mat-tek) overnight. Growth medium was replaced with phenol-red free NSC medium. Multiple full-thickness z-stacks were obtained every 10 min using a Leica SP8 microscope in a $37^{\circ} \mathrm{C}$ chamber supplemented with $5 \% \mathrm{CO}_{2}, 95 \%$ humidity using a 20X/0.8NA objective lens. Time-lapse frames were subsequently analyzed by LasX software.

$\underline{\text { Sorting of tumor cells from co-cultures }}$

Astrocytes were harvested from flasks by Accutase treatment and stained with a 1:1000 dilution of CellTrace ${ }^{\mathrm{TM}}$ Violet Cell Proliferation Dye in PBS at $37^{\circ} \mathrm{C}$ for 20 minutes. Tumor cells and astrocytes were co-cultured at a 1:1 ratio for 48 hours in NSC media. Samples were sorted into RPMI with 20\% FBS and cultured overnight in complete RPMI for subsequent functional assays:

\section{In vitro limiting-dilution assay}

Sorted tumor cells were cultured at decreasing cell densities (400-25 cells/well of a 96-well plate) over 12 technical replicates in Neurobasal medium: Neurobasal Medium (Thermo Fisher Scientific) with 2\% B27 (Thermo Fisher Scientific), 1\% Pen/Strep, $1 \mathrm{mM}$ sodium pyruvate (Thermo Fisher Scientific), 2 mM L-glutamine (Thermo Fisher Scientific), $20 \mathrm{ng} / \mathrm{mL}$ EGF, and 20 $\mathrm{ng} / \mathrm{mL}$ FGF-2, and the number of wells containing spheres was counted after 14 days. The online ELDA tool (http://bioinf.wehi.edu.au/software/elda/) was used to calculate stem cell frequency ${ }^{2}$.

In vivo limiting-dilution assay

Sorted mKate ${ }^{+}$and mKate2- SB28 cells were harvested with Accutase after overnight post-sort culture, pelleted by centrifugation and resuspended in basal RPMI. Cells were counted with trypan blue using a TC-20 cell counter (BioRad) and volume adjusted to achieve decreasing cell concentrations $(18,000-3,000)$ for intracranial implantation. After any volume adjustments, cells were recounted immediately prior to implantation to ensure accurate counts. Subsequently, 4-6-week-old C57BL/6 mice were intracranially implanted with equal numbers of either mKate $2^{+}$ or mKate2- SB28 cells as described above. The identity of the implanted cells in mice was then 
blinded to investigators, who monitored animals daily for neurological symptoms indicative of tumor-related morbidity endpoint.

\section{Seahorse assay}

A total of 15,000-25,000 astrocytes, $\mathrm{mKate}^{+}$and mKate2- tumor cells were seeded in Seahorse XFe24 Cell Culture Microplates (Agilent) in $250 \mu \mathrm{L}$ complete RPMI overnight. The media was replaced with phenol red-free RPMI supplemented with $10 \mathrm{mM}$ glucose, $2 \mathrm{mM}$ glutamine and $1 \mathrm{mM}$ pyruvate (Agilent) and incubated for $45 \mathrm{~min}$ in a non-CO2 incubator. The oxygen consumption rate (OCR) and extracellular acidification rate (ECAR) were measured using the Seahorse XF24 platform (Agilent).

\section{MitoTracker and MitoStatus staining}

CellTrace Violet-stained astrocytes and tumor cells were co-cultured in 6-well plates at a 2:1 ratio for 48 hours. Samples were removed with Accutase. Cells were washed with PBS and stained with MitoStatus Red (BD Biosciences, 1:20,000 dilution) or MitoTracker Deep Red (Thermo Fisher Scientific, 1:100,000 dilution) in complete RPMI 1640 supplemented with 10\% FBS and $1 \%$ Pen/Strep for 15 minutes at $37^{\circ} \mathrm{C}$. Samples were washed with complete RPMI 1640 twice and resuspended in PBS. Sample acquisition was performed using a BD LSRII Fortessa.

Metabolic Flow

The metabolic flow panel was adapted from Ahl et al. ${ }^{3}$. CellTrace Violet-stained astrocytes were co-cultured with tumor cells overnight at a 1:1 ratio. Samples were fixed in eBioscience ${ }^{\mathrm{TM}}$ FoxP3 Transcription Factor Fixation Buffer for 30 minutes on ice and stained with the following antibodies in $1 \mathrm{x}$ Permeabilization Buffer for 30 minutes at room temperature: antiargininosuccinate synthetase 1 (ASS1) [2B10] (Abcam, ab124465, 1:200), anti-ATP synthase F1 subunit alpha (ATP5A) [7H10BD4F9] (Abcam, ab110273, 1:1000), anti-glucose transporter 1 (GLUT1) [EPR3915] (Abcam, ab115730, 1:40), anti-isocitrate dehydrogenase 2 (IDH2) [EPR7577] (Abcam, ab131263, 1:100), anti-glucose 6 phosphate dehydrogenase (G6PD) [EPR20668] (Abcam, ab210702, 1:400), anti-Acetyl-CoA carboxylase (ACC1) [EPR23235-147] (Abcam, ab269273, 1:1000), anti-peroxiredoxin 2 (PRDX2) [EPR5154] (Abcam, ab109367, 1:200), anti-hexokinase 1 (HK1) [EPR10134(B)] (Abcam, ab150423, 1:20), anti-Carnitine palmitoyltransferase I (CPT1A) [EPR21843-71-2F] (Abcam, ab234111, 1:20), anti-SLC20A1 (Thermo Fisher Scientific, 12423-1-AP, 1:200), anti-mouse IgG1, kappa monoclonal [MOPC21] (Abcam, ab18443, 1:200), anti-mouse IgG2b, kappa monoclonal [7E10G10] (Abcam, ab170192, 1:1000) and Rabbit IgG, monoclonal [EPR25A] (Abcam, ab172730, 1:20-1:500). Samples were washed and resuspended in 1x Permeabilization Buffer containing a 1:2000 dilution of Goat AntiMouse IgG H\&L (Alexa Fluor® 647) (Abcam, ab150119) or Donkey Anti-Rabbit IgG H\&L (Alexa Fluor ${ }^{\circledR}$ 647) (Abcam, ab150075). After 30 minutes of incubation at room temperature, cells were washed with permeabilization buffer and resuspended in PBS for analysis with a BD LSRII Fortessa. Geometric mean fluorescence intensity was used to calculate expression levels after subtraction of the background levels from isotype control staining.

Human tumor cell and astrocyte maintenance and culture

Patient-derived xenograft (PDX) D456 was provided by Dr. Darrel Bigner at Duke University, and PDX JX22 was provided by Dr. Jann Sarkaria at the Mayo Clinic. DMEM:F12 containing $10 \mathrm{ng} / \mathrm{mL}$ EGF, $10 \mathrm{ng} / \mathrm{mL}$ FGF, 1\% sodium pyruvate, 2\% GEM21 (Gemini Bio), and $1 \%$ Pen/Strep was used to culture the PDX lines. A clone of HEK293T cells able to grow in serumfree media (CSC293T) was generated and cultured as previously described ${ }^{4}$. Normal human 
astrocytes (NHAs; provided by Dr. Russell Pieper at UCSF) were cultured in DMEM supplemented with 10\% FBS, 1\% N2 NeuroPlex Supplement (Gemini Bio), 3 ng/mL EGF, and $1 \%$ Pen/Strep. CSC293T cells were transfected with psPAX2, pCMV-VSVG, and pLYS1-MitoGFP or pCMV-RFP using FuGENE HD transfection reagent (Promega). Viral concentration was determined using the Lenti-X qRT-PCR Titration kit (Takara Bio). NHAs were transduced with mito-GFP lentivirus and selected for cells stably expressing mito-GFP using puromycin. D456 and JX22 cells were infected with RFP lentivirus and selected using Blastacidin S (Gibco) to select for stable RFP-expressing cells. Where indicated, cells were sorted for mito-GFP ${ }^{+}$or $\mathrm{RFP}^{+}$with assistance from the Flow Cytometry Core at the University of Alabama at Birmingham.

ImageStream

RFP-expressing D456 or JX22 cells were co-cultured at a 1:1 ratio in the presence of mito$\mathrm{GFP}^{+}$NHAs for 24hrs. Samples were imaged at 40x magnification and extended depth of field (EDF). Mito-GFP was acquired on ch02 and RFP on ch04. Ch01 and ch09 are used for brightfield imaging, and ch12 was used for side scatter. A total of 5000 events was recorded, and relevant single color and unstained controls were used. Data was analyzed using IDEAS software (version 6.2; EMD Millipore).

\section{$\underline{\text { RNA Sequencing }}$}

mKate $^{+}$and mKate SB28 cells and astrocytes from 3 distinct co-cultures (biological replicates) were sorted into multiple $1.5 \mathrm{~mL}$ DNA LoBind microtubes (Eppendorf) each containing $700 \mu \mathrm{L}$ of RLT Plus lysis buffer (Qiagen) supplemented with 1\% 2-mercaptoethanol. RNA isolation was performed using the RNEasy Plus Micro kit (Qiagen). Briefly, sorted cell microtubes were brought to room temperature and vortexed vigorously. Cells were further lysed by passing the cell suspension 5 times through a $20 \mathrm{G}$ needle. Downstream isolation was performed per manufacturer's protocol with the following exception. Up to $1400 \mu \mathrm{L}$ of sorted cell suspension was sequentially loaded onto each DNA binding column. When multiple columns were used for a given sample, elution of DNA was performed sequentially, using $15 \mu \mathrm{L}$ of RNAse-free water to pool the isolated DNA in a minimal volume.

RNA sequencing and analysis were performed by GENEWIZ (South Plainfield, NJ). Briefly, samples were sequenced using an Illumina HiSeq, $2 \times 150$ bp configuration and $\geq 350 \mathrm{M}$ raw paired-end reads. An average of $41.6 \mathrm{M}$ paired-end reads was sequenced across 9 samples. After Illumina universal adapters were trimmed, the reads were mapped to the Mus musculus GRCm38 reference genome using the STAR aligner v.2.5.2b. Unique gene hit counts were calculated by using featureCounts from the Subread package v.1.5.2.

For comparison of tumor cells with astrocytes, genes with an adjusted p-value $<0.05$ and absolute $\log 2$ fold change $>1$ were called as differentially expressed genes using DESeq2. For assessment of differentially up-regulated pathways in mKate ${ }^{+}$versus mKate ${ }^{-}$SB28 cells, genes that were up-regulated $>1.5$-fold with a count number of $>50$ and an unadjusted p-value of $<0.05$ (table S1) were plugged into https://maayanlab.cloud/Enrichr/.

\section{Protein-protein interactions and network visualization}

Differential expression analysis was performed using edgeR $3.34^{5}$. Genes with count per million greater than 1 in at least 2 samples were used for the analysis. $\mathrm{P}$ values $<0.05$ were considered significant. The mouse differentially expressed genes (DEGs) were mapped to the human homologs using the NCBI HomoloGene database (https://www.ncbi.nlm.nih.gov/homologene). We then performed the enrichment analysis using Enrichr ${ }^{6}$ for the entire DEGs and for the up- and down-regulated genes separately. 
The protein-protein interactions (PPIs) among the DEGs were extracted using a human protein interactome we built previously ${ }^{7}$ that contains 17,706 protein nodes and 351,444 PPI edges. We then visualized this protein-protein interaction network using Cytoscape $3.8^{8}$. Genes that localize to mitochondria are indicated by diamond node shape based on the Human MitoCarta2.0 database ${ }^{9}$.

\section{Data representation and analysis}

Flow cytometry data were analyzed and generated using FlowJo software (BD Biosciences, v10.7.2). Graphs were generated and statistical analysis were performed using Excel (Microsoft Office, v16.52) or Prism (GraphPad, v9.2.0) software. All measurements shown represent distinct samples, unless otherwise indicated. All statistical tests are 2-tailed and corrected for multiple comparisons, unless otherwise indicated.

1 Marshall, G. P., 2nd, Demir, M., Steindler, D. A. \& Laywell, E. D. Subventricular zone microglia possess a unique capacity for massive in vitro expansion. Glia 56, 1799-1808, doi:10.1002/glia.20730 (2008).

$2 \mathrm{Hu}, \mathrm{Y}$. \& Smyth, G. K. ELDA: extreme limiting dilution analysis for comparing depleted and enriched populations in stem cell and other assays. J Immunol Methods 347, 70-78, doi:10.1016/j.jim.2009.06.008 (2009).

3 Ahl, P. J. et al. Met-Flow, a strategy for single-cell metabolic analysis highlights dynamic changes in immune subpopulations. Commun Biol 3, 305, doi:10.1038/s42003-020-10279 (2020).

$4 \quad$ Walker, K. \& Hjelmeland, A. Method for Efficient Transduction of Cancer Stem Cells. $J$ Cancer Stem Cell Res 2, doi:10.14343/JCSCR.2014.2e1008 (2014).

5 Robinson, M. D., McCarthy, D. J. \& Smyth, G. K. edgeR: a Bioconductor package for differential expression analysis of digital gene expression data. Bioinformatics 26, 139140, doi:10.1093/bioinformatics/btp616 (2010).

$306 \quad$ Kuleshov, M. V. et al. Enrichr: a comprehensive gene set enrichment analysis web server 2016 update. Nucleic Acids Res 44, W90-97, doi:10.1093/nar/gkw377 (2016).

7 Cheng, F. et al. Network-based approach to prediction and population-based validation of in silico drug repurposing. Nat Commun 9, 2691, doi:10.1038/s41467-018-05116-5 (2018).

358 Shannon, P. et al. Cytoscape: a software environment for integrated models of biomolecular interaction networks. Genome Res 13, 2498-2504, doi:10.1101/gr.1239303 (2003).

9 Calvo, S. E., Clauser, K. R. \& Mootha, V. K. MitoCarta2.0: an updated inventory of mammalian mitochondrial proteins. Nucleic Acids Res 44, D1251-1257, doi:10.1093/nar/gkv1003 (2016). 
Acknowledgments: The authors would like to thank Dr. Erin Mulkearns-Hubert for editorial assistance and Ms. Amanda Mendelsohn for illustrations. We would like to thank the Lathia laboratory, LRI Flow Cytometry Core, Imaging Core, Dr. John Peterson, Dr. Anny Mulla and Dr. Prasad Sathyamangla for their technical assistance and discussions.

Author contributions:

Conceptualization: DCW, DB, JDL

Methodology: DCW, DB, SW, AL, YZ

Investigation: DCW, DB, SW, AL, YZ, GD, JS

Visualization: DCW, DB, SW, YZ

Funding acquisition: DCW, DB, JDL

Project administration: JDL

Supervision: JAM, FC, ABH, JDL

Writing - original draft: DCW, DB, JDL

Writing - review \& editing: DCW, DB, SW, AL, GD, JS, JAM, YZ, FC, ABH, JDL

\section{Funding:}

Lerner Research Institute, Case Comprehensive Cancer Center (JDL)

VeloSano Cancer Research Pilot Award (DCW, DB, JDL)

Clinical and Translational Science Collaborative of Cleveland, UL1TR002548 from the National Center for Advancing Translational Sciences (NCATS)

2020 VeloSano Trainee Dream Experiment (DCW)

National Institutes of Health grants 5T32AI007024 and 5TL1TR002549 (DCW)

National Institutes of Health grant K99CA248611 (DB)

National Institutes of Health grant F30CA250254 (AL)

This work utilized the Leica SP8 confocal microscope that was purchased with funding from National Institutes of Health SIG grant 1S10OD019972-01

Competing interests: The authors declare that they have no competing interests.

Data and materials availability: Sequencing files have been deposited to GEO with the accession number GSE183004. All other data are available in the main text or the supplementary materials.

\section{Supplementary Materials}

Supplementary Text

Figs. S1 to S11

Tables S1 


\section{Supplementary Materials for}

\section{Brain cells donate mitochondria to increase glioblastoma tumorigenicity}

Dionysios C. Watson ${ }^{1,2,3,4 \dagger}$, Defne Bayik ${ }^{1,2 \dagger}$, Sarah E. Williford ${ }^{5}$, Adam Lauko ${ }^{1,4,6}$, Yadi Zhou ${ }^{1}$, Gauravi Deshpande ${ }^{1}$, Juliana Seder ${ }^{7}$, Jason A. Mears ${ }^{2,4}$, Feixiong Cheng ${ }^{1,2}$, Anita B. Hjelmeland $^{5}$, Justin D. Lathia ${ }^{1,2^{*}}$

Correspondence to: 1athiaj@ccf.org

This PDF file includes:

Supplementary Text

Figs. S1 to S11

Tables S1

Captions for Movies S1

Other Supplementary Materials for this manuscript include the following:

Movies S1 


\section{Supplementary Text}

3D segmentation algorithm parameters (GL261)

(1) Define GL261 objects:

a. Channel 3 (GFP) threshold

i. intensities: 11-185

ii. Minimum object size $=10000$ cubic microns

b. Fill holes in objects

c. Separate touching objects

i. Object size guide $=1200$ cubic microns

(2) Define mitochondria objects

a. Channel 1 (mito::mKate2) threshold

i. Intensities 20-255

ii. Minimum object size $=1$ cubic micron

b. Separate touching objects

i. Object size guide $=25$ cubic microns

c. Filter population

i. Volume $>=0.1$ cubic microns

(3) Compartmentalize mitochondria objects inside GL261 objects

3D segmentation algorithm parameters (SB28)

(1) Define SB28 objects:

a. Channel 3 (GFP) threshold

i. intensities: $20-255$

ii. Minimum object size $=10000$ cubic microns

b. Fill holes in objects

c. Separate touching objects

i. Object size guide $=1200$ cubic microns

d. Filter population

i. Volume $>600$ cubic microns

(2) Define mitochondria objects

a. Channel 1 (mito::mKate2) threshold

i. Intensities 20-255

ii. Minimum object size $=1$ cubic micron

b. Separate touching objects

i. Object size guide $=25$ cubic microns

c. Filter population

i. Volume $>=0.1$ cubic microns

(3) Compartmentalize mitochondria objects inside SB28 objects 

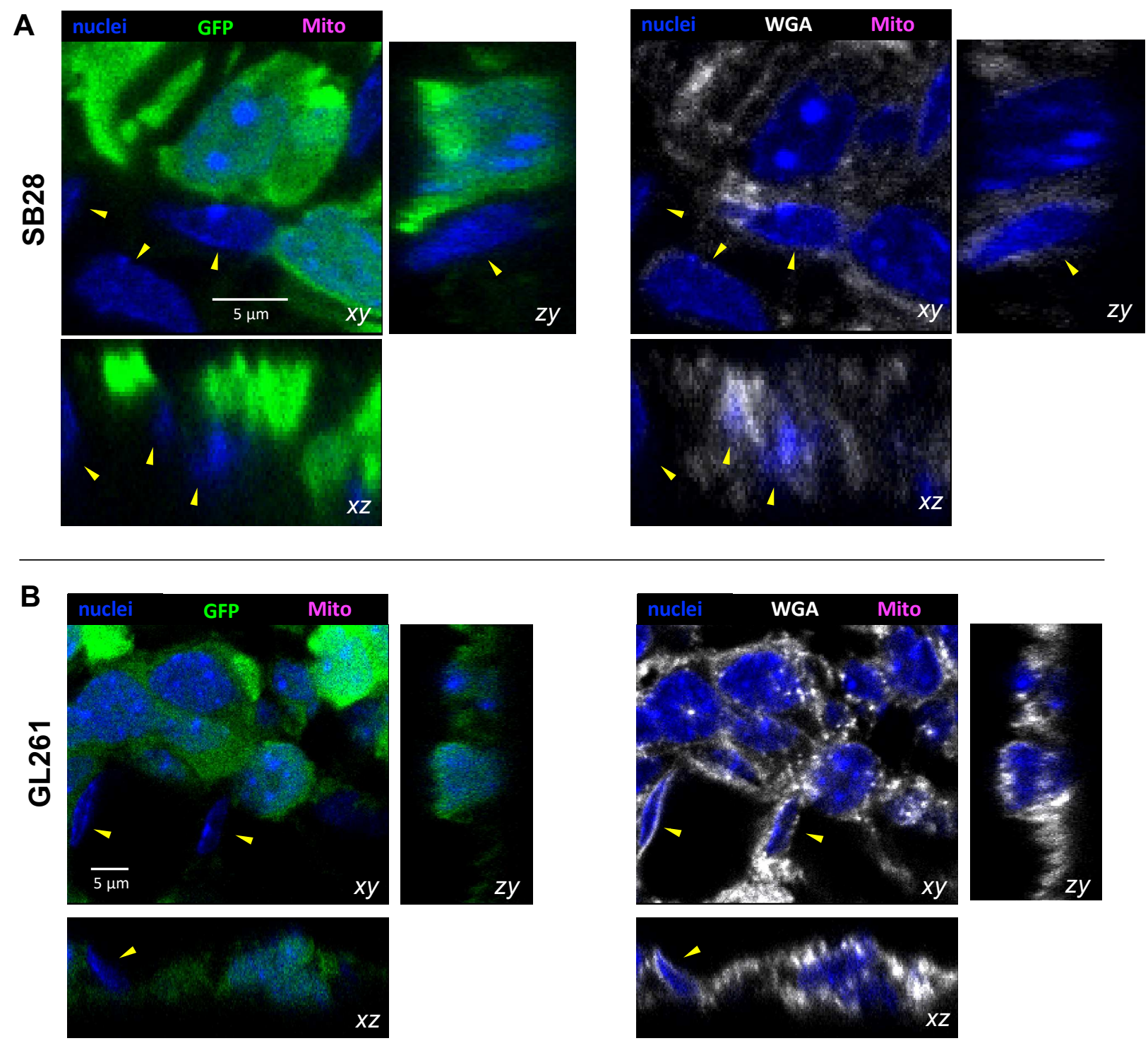

\section{$y$}

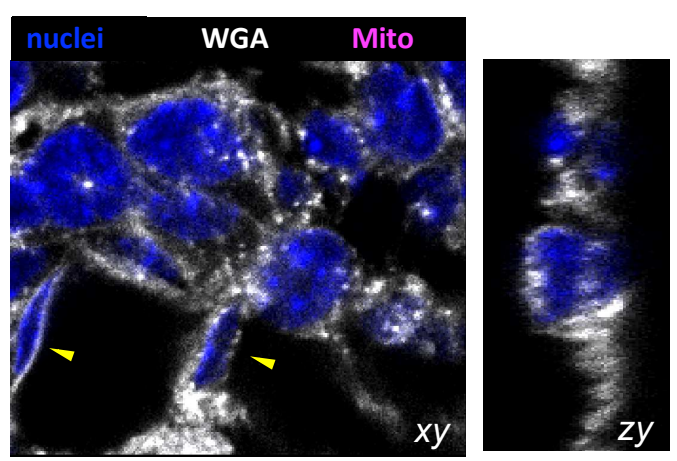

Fig. S1: Tumor-host cell interface in brain tumor sections of wildtype mice. GFP-expressing GBM tumor cell lines were implanted intracranially into wildtype C57BL/6 mice. Formalin-fixed, permeabilized cryosections from mouse GBM tumors were stained with anti-GFP, wheat germ agglutinin (WGA) and Hoechst 33342 nuclear stain. Confocal z-stacks were acquired with the detection of the above fluorescent signals, in addition to the mito::mKate 2 signal to assess the level of mKate2 background signal at the tumor:host cell interface. Close-up single focal planes and zstack orthogonal reconstructions of (A) SB28 and (B) GL261 tumor cells (green) adjacent to host cells (yellow arrowheads). 


\section{A1}

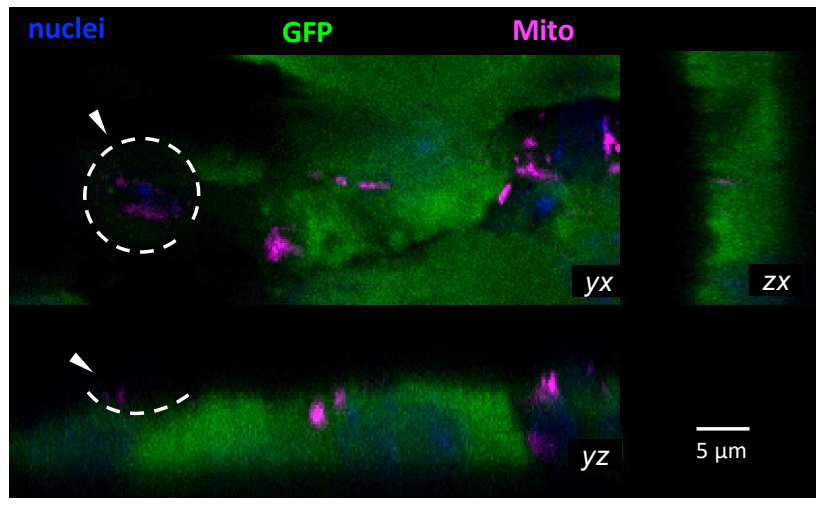

B1

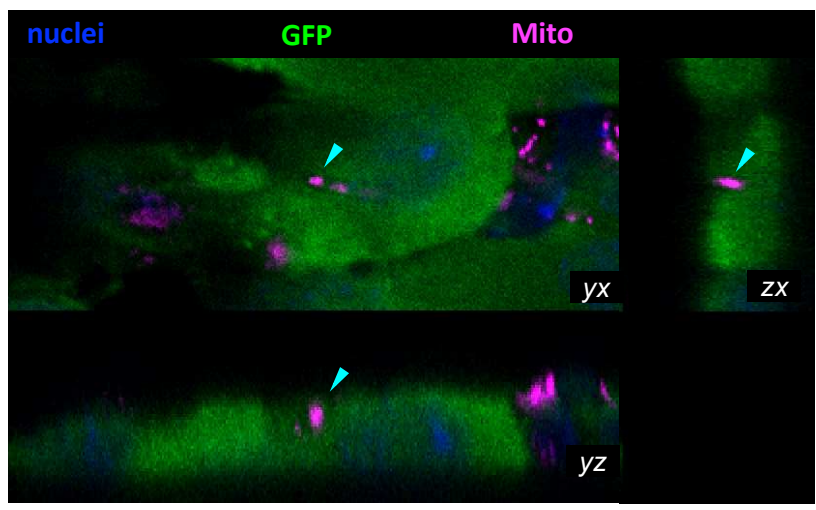

C1

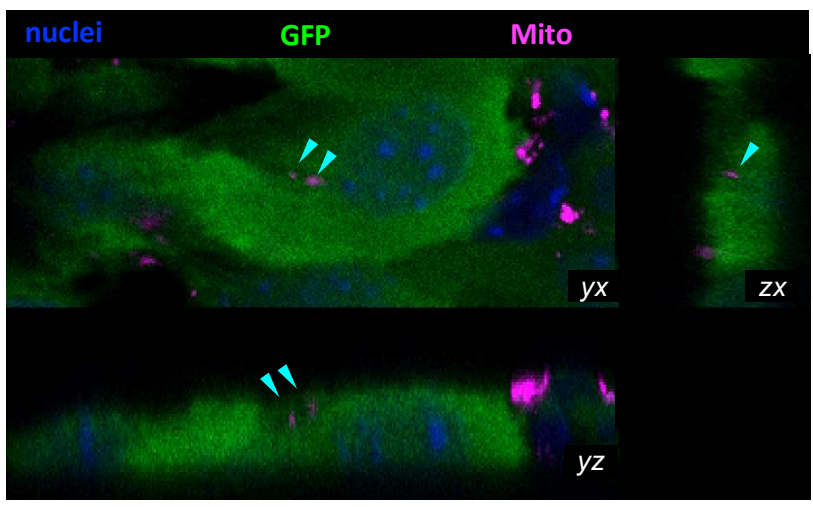

A2

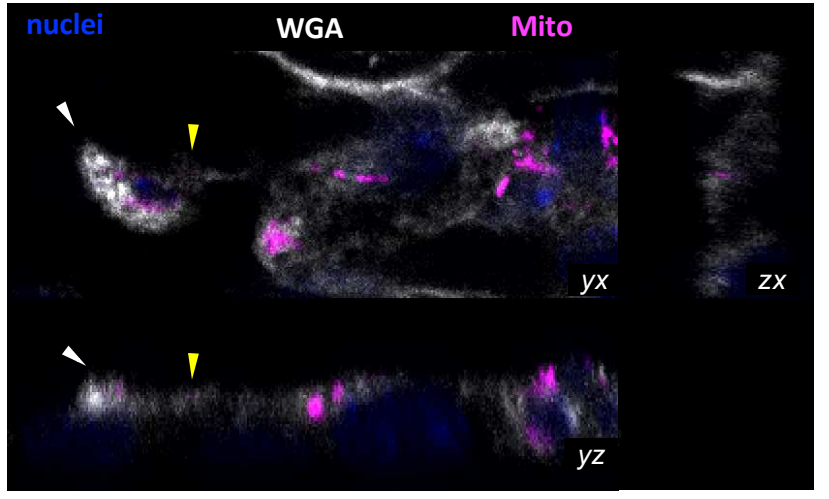

B2

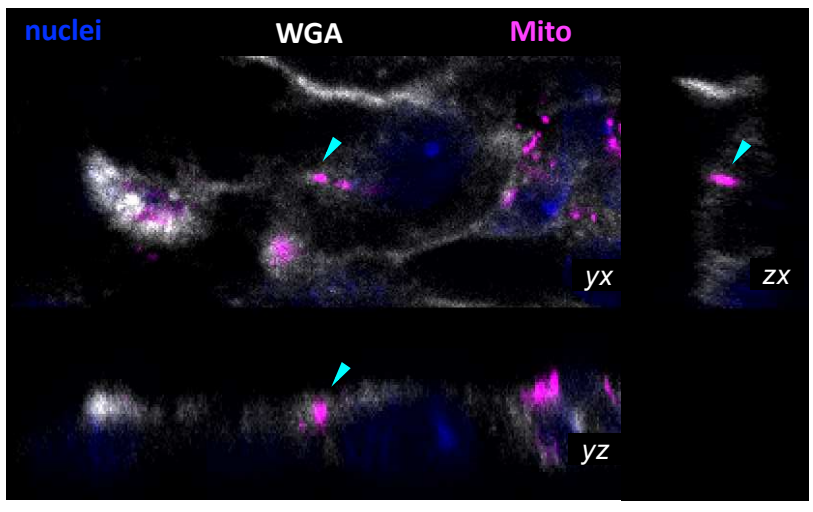

C2

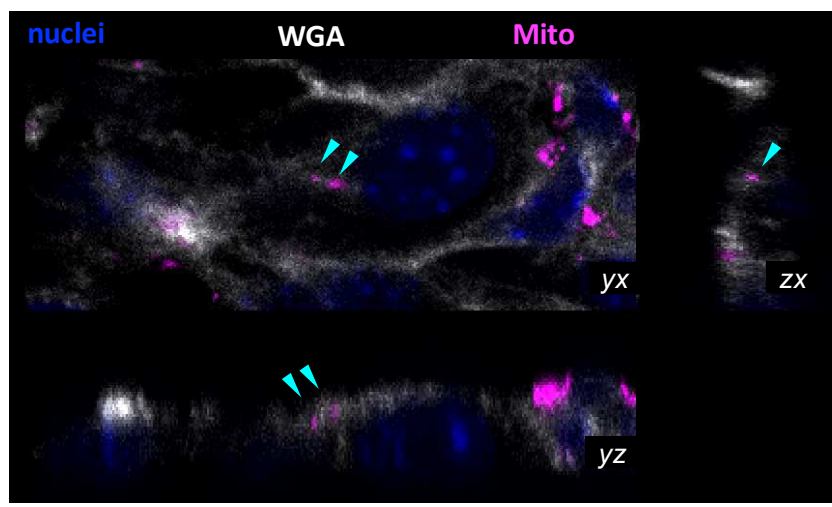

\section{Legend}

$\square$ Potential mitochondria source host cell

$\square$ Host mitochondria within intercellular connection

$\square$ Host mitochondria completely within GFP+ tumor cell cytoplasm

Fig. S2: Transfer of mitochondria to SB28 cells in vivo. $(A \rightarrow C)$ Sequential confocal planes $(y x)$ and accompanying orthogonal reconstructions (zx, yz) of SB28 GBM tumors in mice, demonstrating an intercellular connection between a mito::mKate $2^{+}$host cell (white arrowheads) and $\mathrm{GFP}^{+}$tumor cell. Host $\mathrm{mKate} 2^{+}$mitochondria within the intercellular connection are indicated 
by yellow arrowheads. The mitochondria at the end of the connection are surrounded by GFP signal, suggesting incorporation into the recipient tumor cell cytoplasm (cyan arrowheads). 

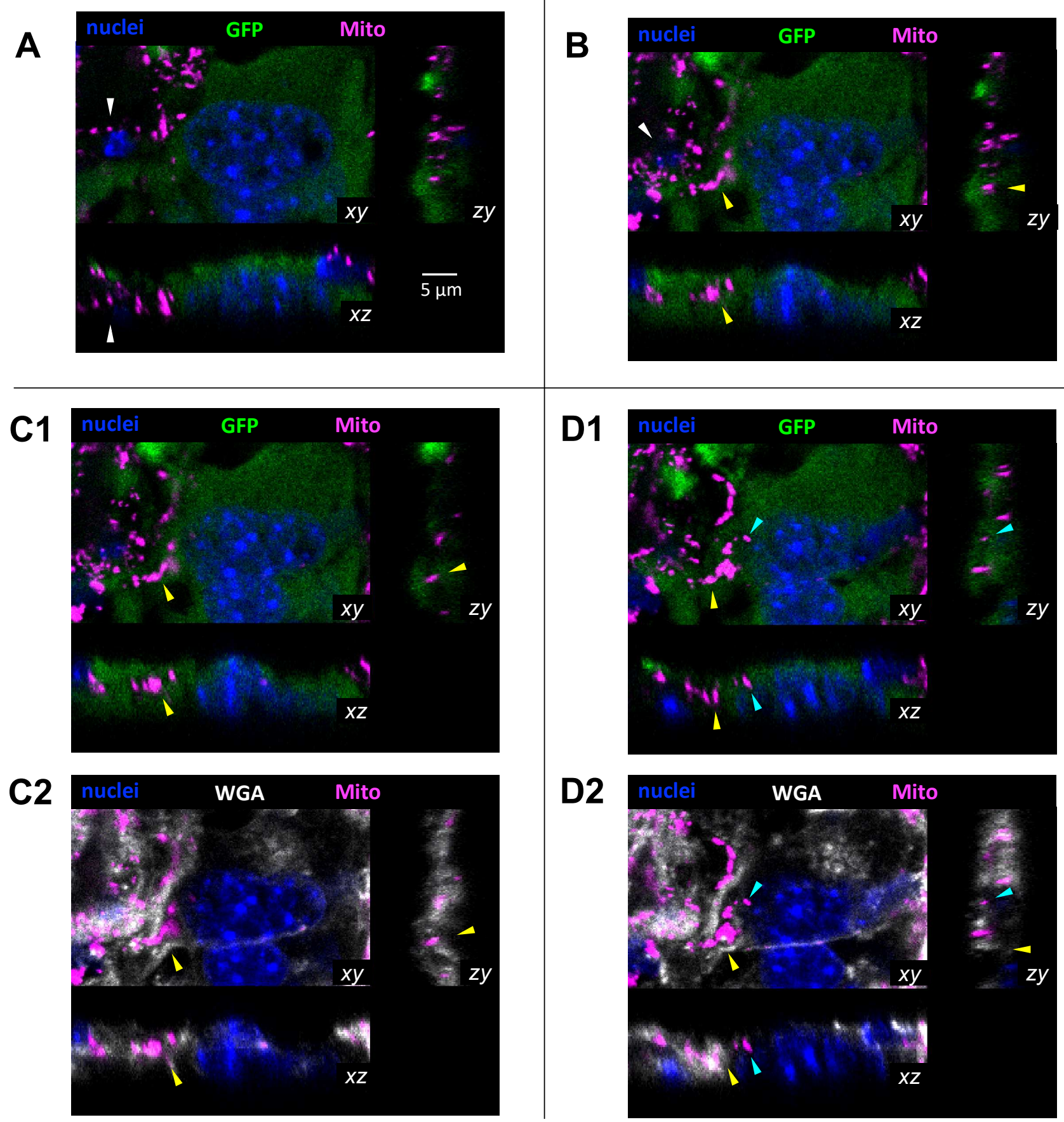

\section{Legend}

$\square$ Potential mitochondria source host cell

$\square$ Host mitochondria within intercellular connection

$\square$ Host mitochondria completely within GFP+ tumor cell cytoplasm

Fig. S3: Transfer of mitochondria to GL261 cells in vivo. $(A \rightarrow D)$ Sequential confocal planes (xy) and accompanying orthogonal reconstructions (zy, xz) of GL261 GBM tumors in mice, demonstrating an intercellular connection between a mito::mKate $2^{+}$host cell (white arrowheads) and $\mathrm{GFP}^{+}$tumor cell. Host $\mathrm{mKate} 2^{+}$mitochondria within the intercellular connection are indicated 
by yellow arrowheads. The mitochondrion at the end of the connection is surrounded by GFP signal, suggesting complete incorporation in the recipient tumor cell cytoplasm (cyan arrowhead). 
A
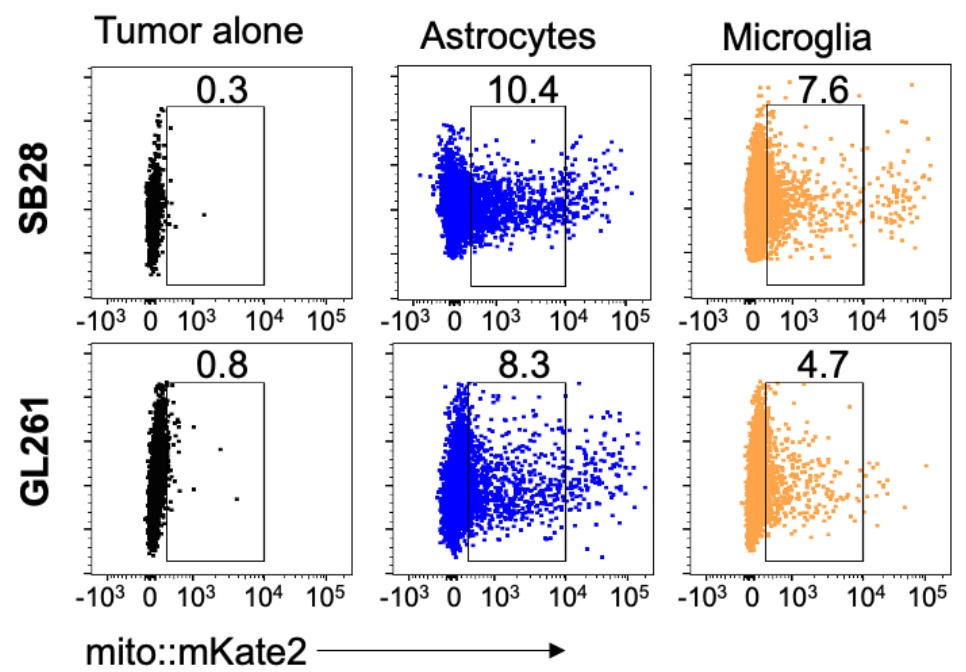

Macrophages
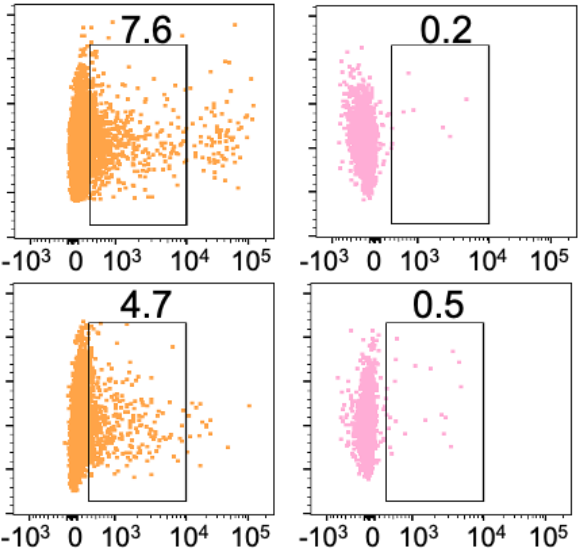

B

C

SB28

GL261
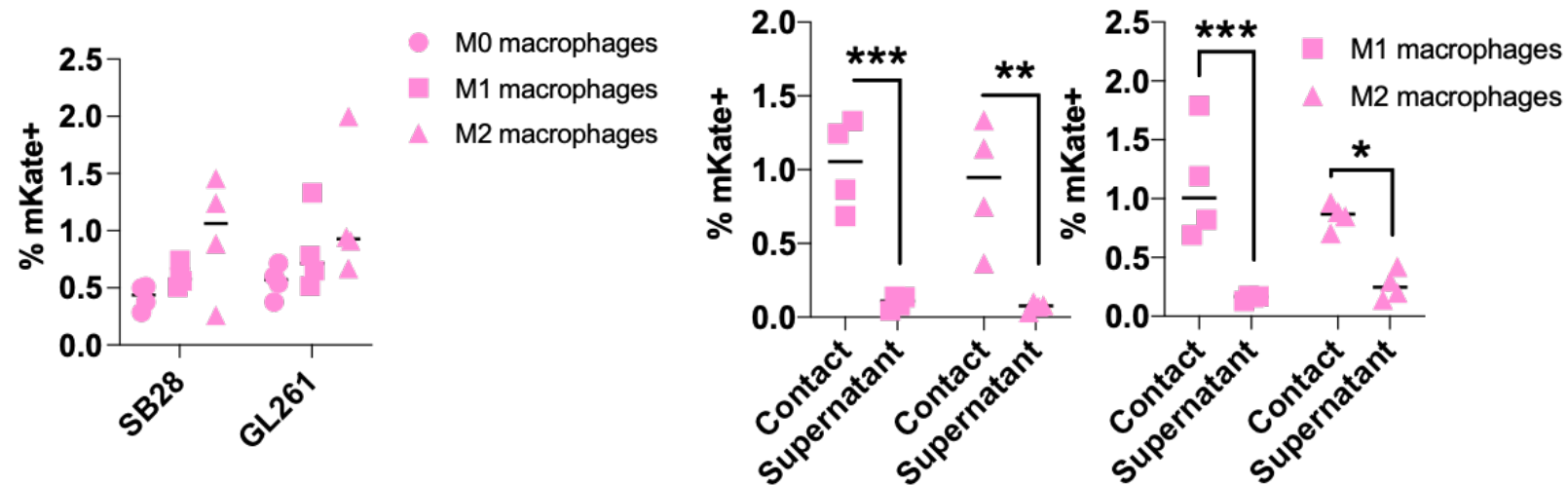

Fig. S4: Macrophages have a limited contribution to mitochondrial uptake by GBM cells. (A) Representative dot plots depicting the frequency of mitochondrial uptake by GBM cells following 2 hours of co-culture with astrocytes, microglia and macrophages at a ratio of 2:1. (BC) Bone marrow cells were cultured with $50 \mathrm{ng} / \mathrm{ml}$ M-CSF for 6 days and treated with $50 \mathrm{ng} / \mathrm{ml}$ IFN $\gamma$ (M1-like) or IL-4 (M2-like) during the last 48 hours. (B) mKate $2^{+} \mathrm{GBM}$ cell frequency was assessed by flow cytometry after 2 hours of co-culture with the denoted macrophage donors. (C) Contact-dependent (contact) and independent (supernatant) mitochondrial transfer was also analyzed by flow cytometry. $\mathrm{n}=4$ biological replicates. ${ }^{*} \mathrm{p}<0.05,{ }^{* *} \mathrm{p}<0.01,{ }^{* * *} \mathrm{p}<0.001,2$-way ANOVA. 


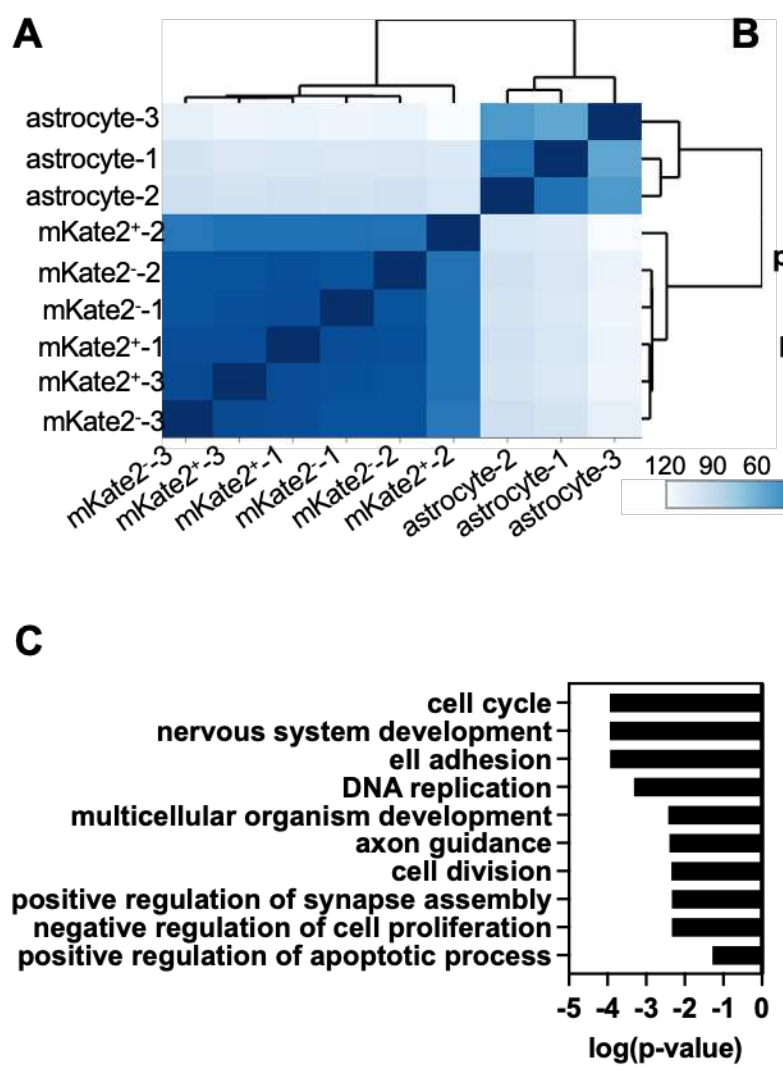

B

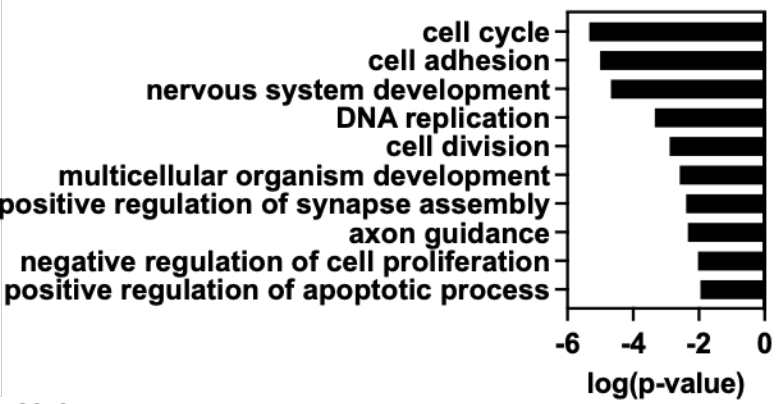

D

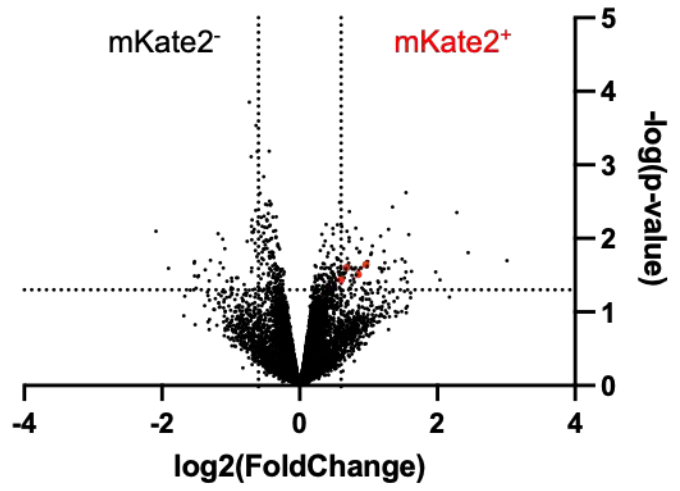

Fig. S5: Sorted $\mathbf{m K a t e} 2^{+}$cells are not contaminated by astrocytes. (A) Heatmap demonstrating separate unsupervised hierarchical clustering of astrocytes from mKate2- and mKate $2^{+}$tumor cells based on gene expression profiling. GO pathway analysis of differentially expressed genes between (B) mKate2 ${ }^{-}$cells and astrocytes and (C) mKate $2^{+}$cells and astrocytes. (D) Volcano plot representing differential gene expression signature of $m K$ ate $2^{+}$versus $m K a t e 2^{-}$cells. Dashed lines mark fold change $>1.5$ and $p$-value $<0.05$. Genes that are mapped to mitochondria-related networks are shown in red. 


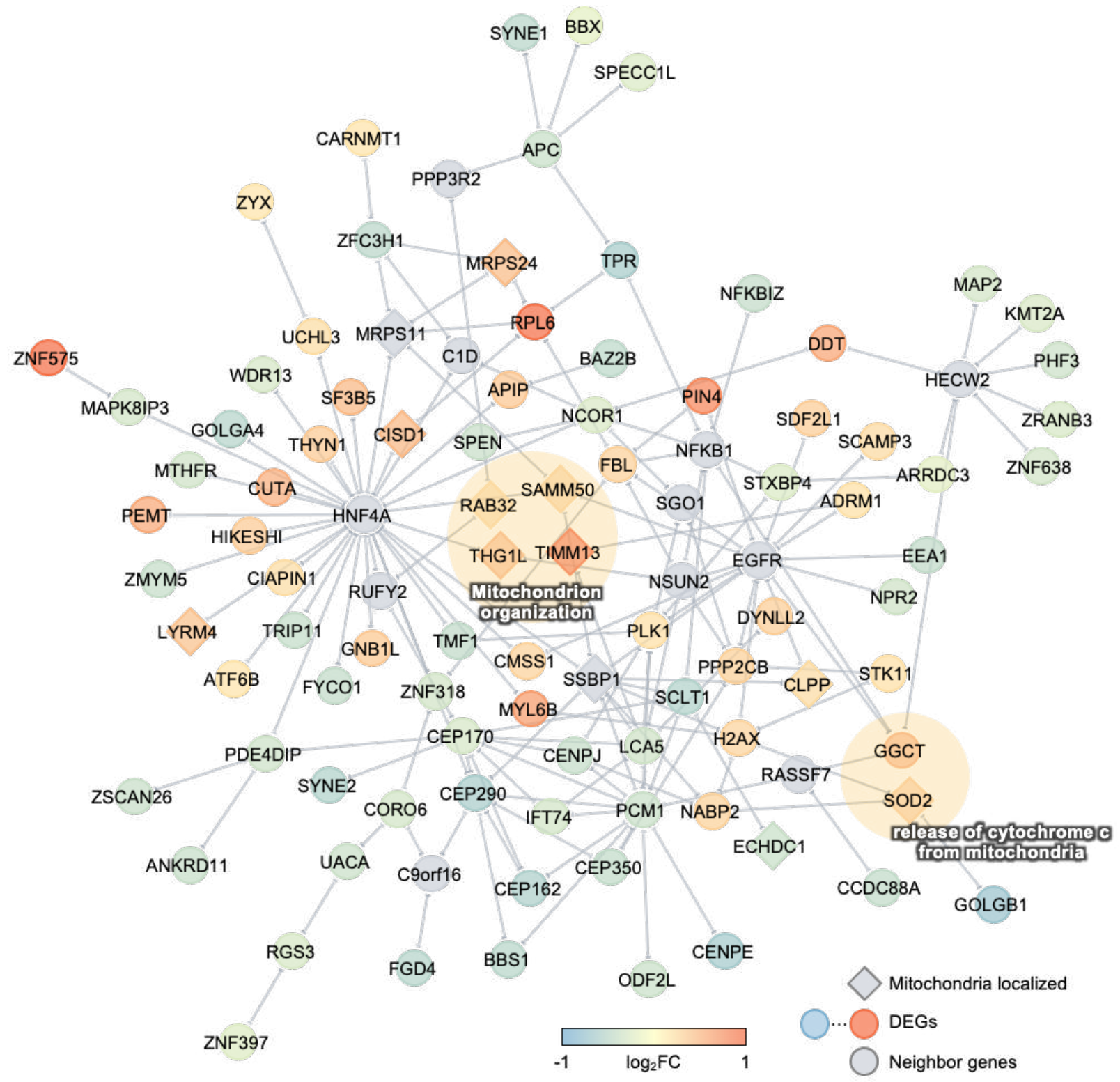

Fig. S6: Differentially up-regulated genes are enriched in the mitochondrial network. Protein-protein interaction network for the differentially expressed genes. Mouse genes were mapped to human genes according to NCBI HomoloGene. Protein-protein interactions were extracted for these genes/proteins using our human protein interactome. Diamond-shaped nodes indicate that these genes are mitochondrially localized based on the Human MitoCarta2.0 database. Node color shows the $\log 2$ fold change of the genes. 

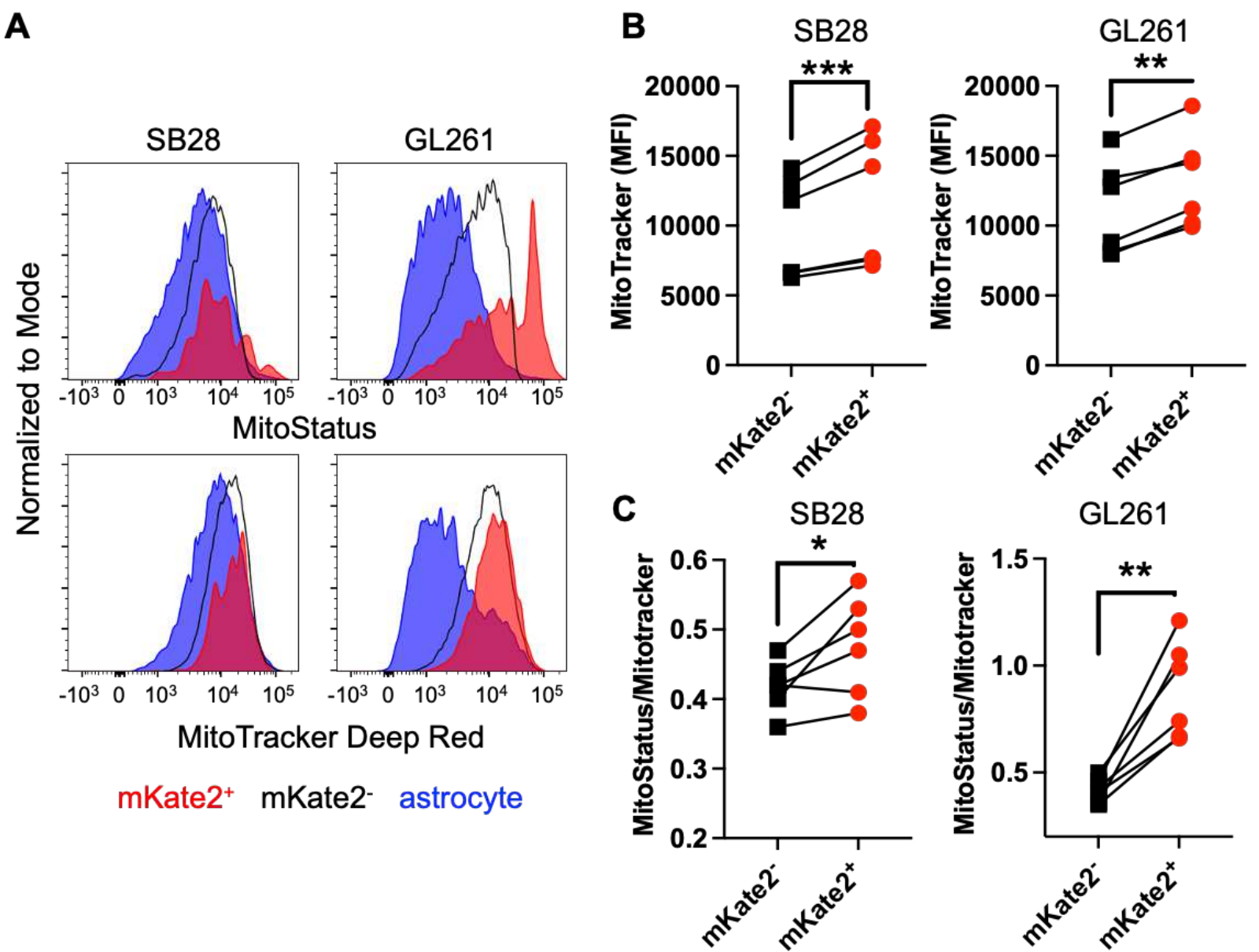

Fig. S7: Acquisition of donor mitochondria leads to higher mitochondrial content and membrane potential. (A) Representative histograms depicting MitoStatus and Mitotracker Deep Red staining of astrocytes and mKate2 ${ }^{+}$and mKate2- SB28 (left) and GL261 (right). (B) Background-corrected Mitotracker Deep Red geometric mean fluorescence of SB28 and GL261 cells revealed an average $15-16 \%$ increase in mitochondrial mass in mKate $2^{+}$compared to mKate2cells. (C) Ratio of MitoStatus to MitoTracker Deep Red staining demonstrated higher membrane potential in $\mathrm{mKate} 2^{+}$cells when normalized to total mitochondrial mass. $\mathrm{n}=6$ biological replicates. $* \mathrm{p}<0.05, * * \mathrm{p}<0.01$ and $* * * \mathrm{p}<0.01$ as determined by paired t-test. 
A Anabolic Pathways

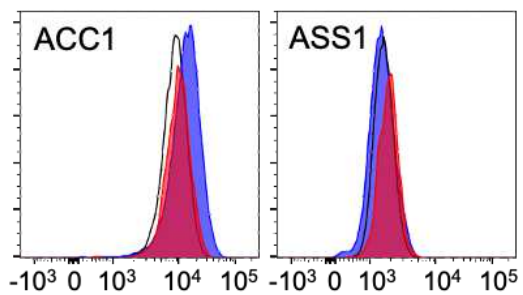

Catabolic Pathways
Metabolic Transporter/Sensor

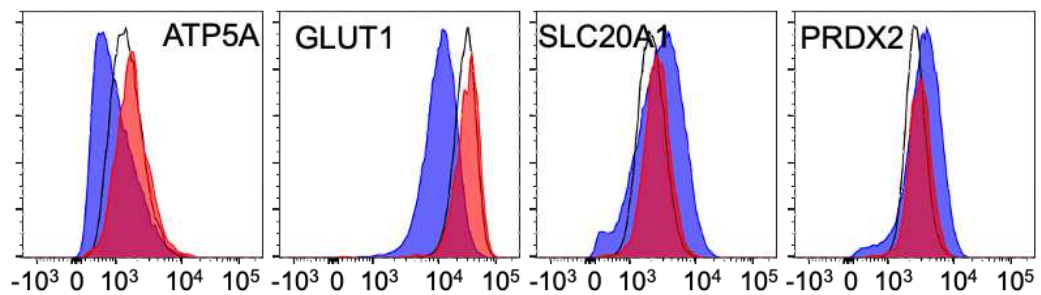

Isotype
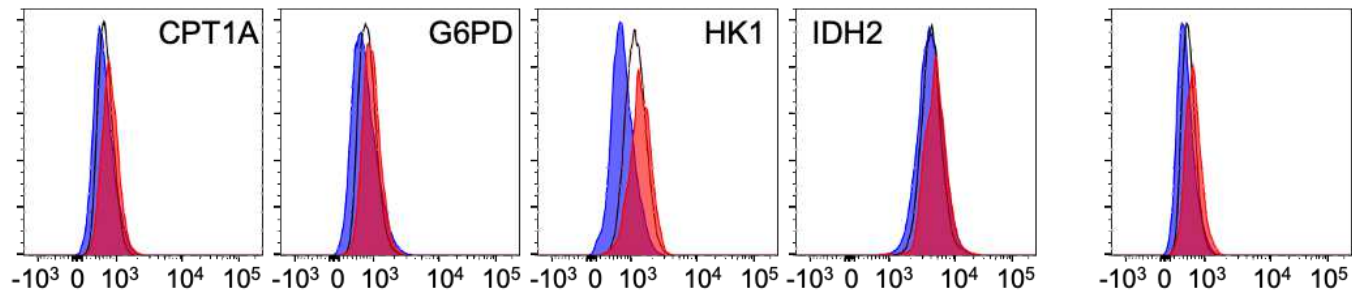

mKate $2^{+}$

mKate2-

astrocyte
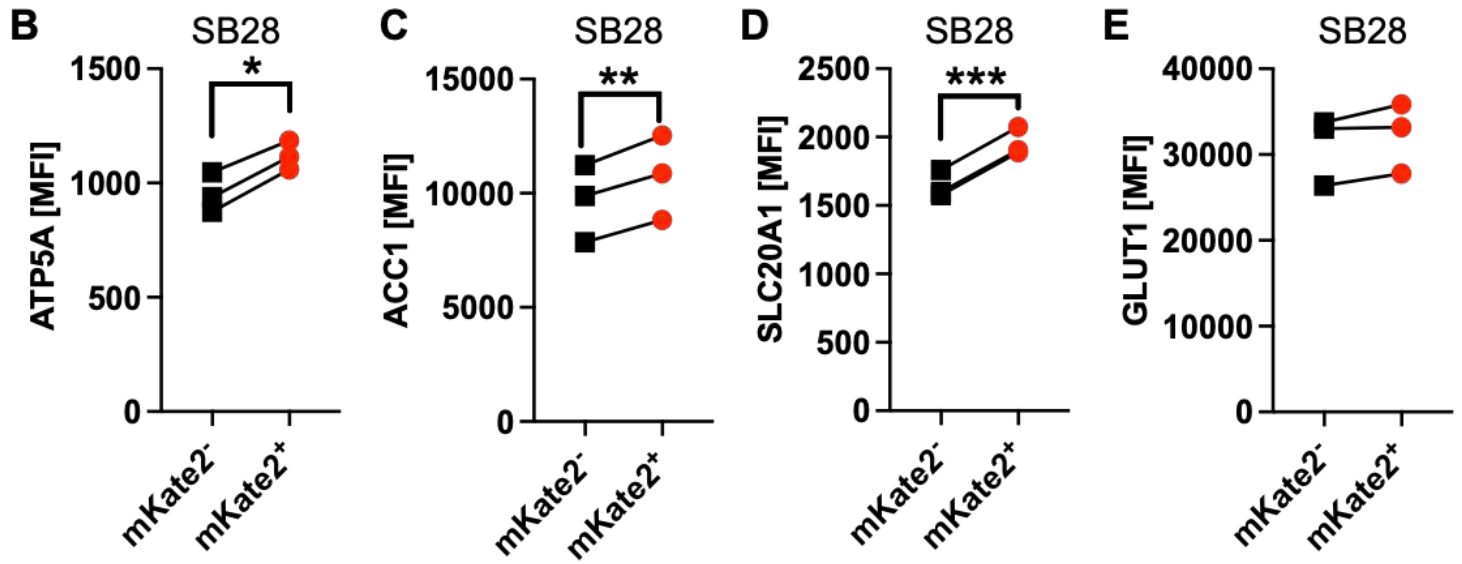

GL261

GL261
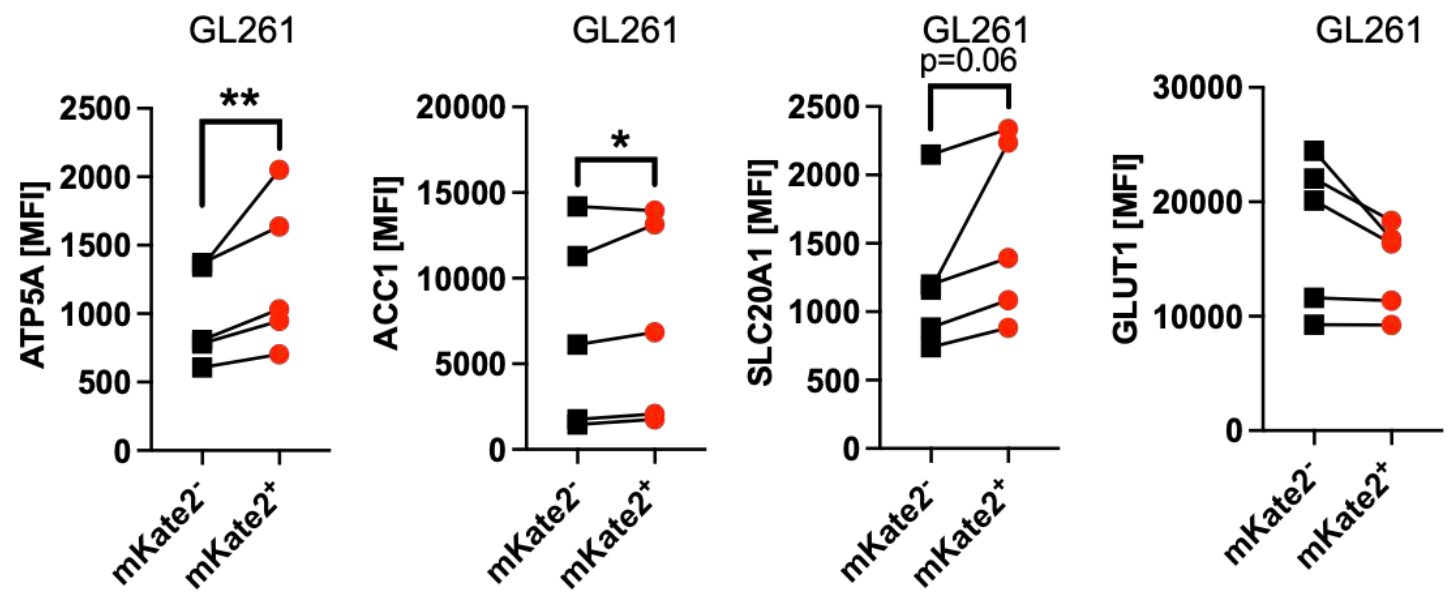

Fig. S8: GBM cells that acquire astrocyte mitochondria have higher levels of metabolic proteins associated with ATP and fatty acid synthesis. GBM cell lines were co-cultured with mito::mKate2+ astrocytes for 24 hours and stained with antibodies against key metabolic proteins, as denoted. Expression levels were assessed by flow cytometry. (A) Representative histograms depicting differential expression of critical metabolic proteins by astrocytes and $\mathrm{mKate} 2^{+}$and mKate2- SB28 cells. Astrocytes had higher levels of acetyl-CoA carboxylase (ACC1), SLC20A1 
and peroxiredoxin-2 (PRDX2), indicative of more oxidative phosphorylation. In contrast, tumor cells had higher levels of glucose transporter 1 (GLUT 1) and hexokinase 1 (HK1), pointing to a more glycolytic metabolism. (B-E) Aggregate data from n=3-5 biological replicates of SB28 (top row) and GL261 (bottom row) cells co-cultured with astrocytes. Panels depict isotype backgroundcorrected, geometric mean fluorescence intensity (MFI) of the expression of the indicated metabolic proteins. ${ }^{*} \mathrm{p}<0.05,{ }^{* *} \mathrm{p}<0.01,{ }^{* * *} \mathrm{p}<0.001$, paired t-test. 


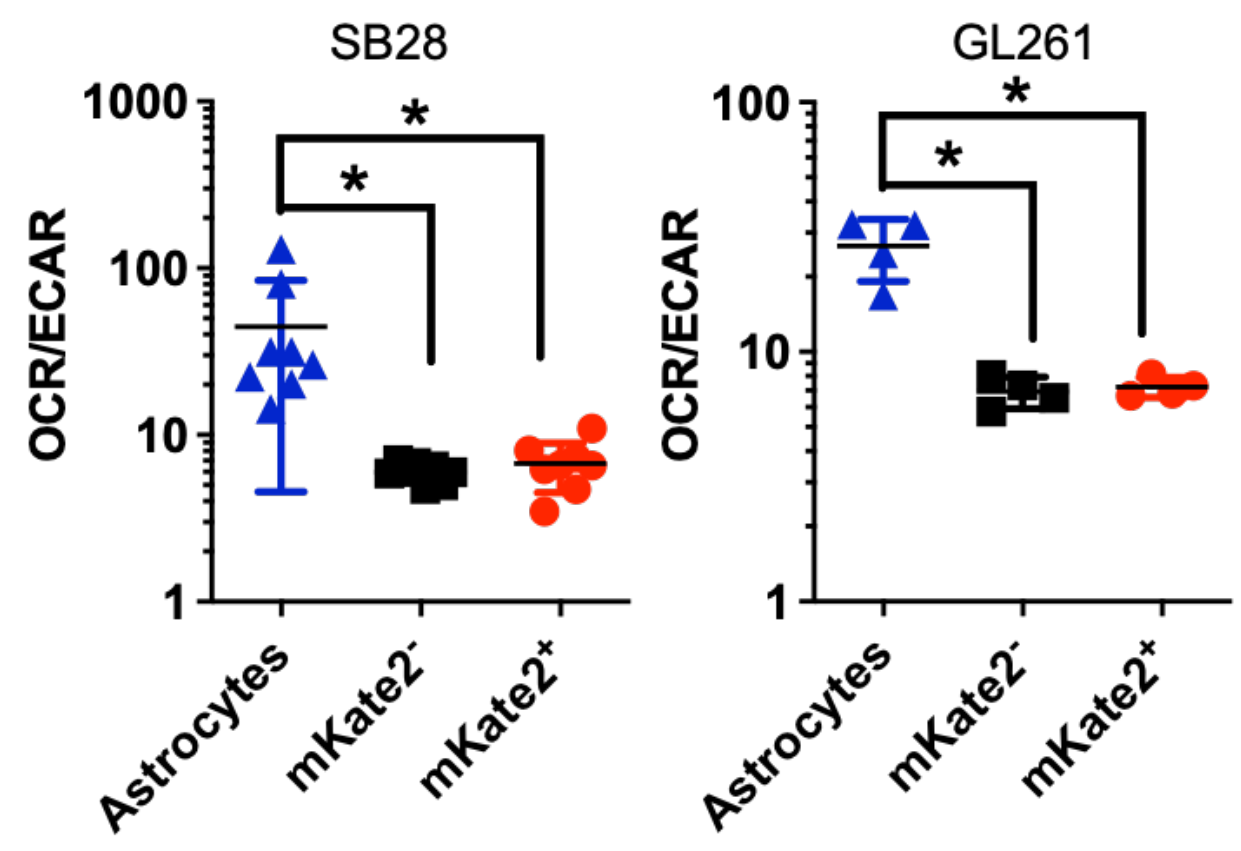

Fig. S9: mKate $^{+}$and mKate2- SB28 cells sorted from astrocyte co-cultures similarly rely on glycolysis in a glucose-rich environment. GBM cells were sorted from mito::mKate $2^{+}$astrocyte co-cultures after $48 \mathrm{~h}$, cultured overnight, and then subjected to Seahorse assay in standard assay media in the presence of excess glucose. Oxygen consumption rate (OCR) and extracellular acidification rate (ECAR) were measured at baseline in $\mathrm{n}=4-8$ biological replicates. ${ }^{*} \mathrm{p}<0.05$ as determined by one-way ANOVA. 


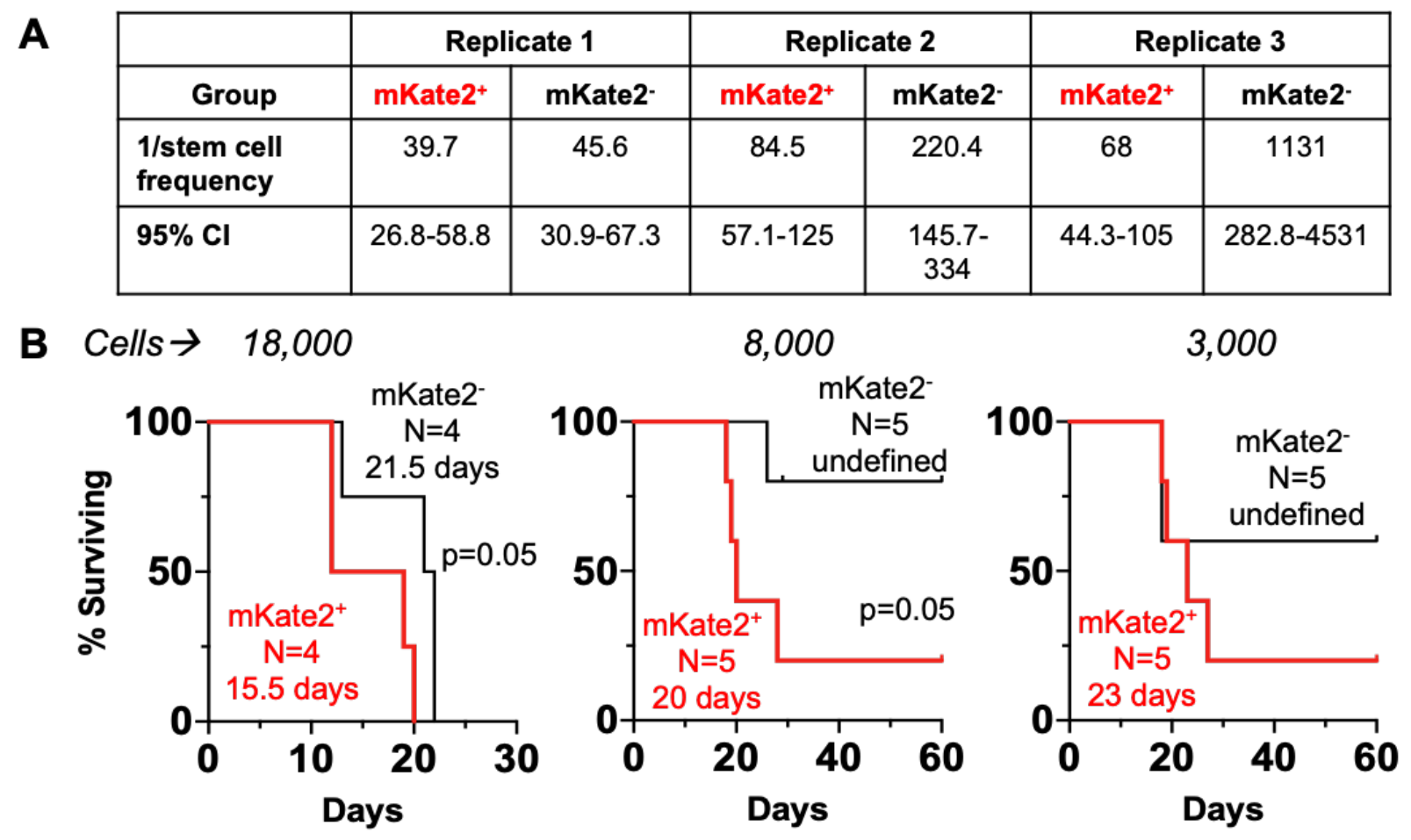

Fig. S10: KKate2 $^{+}$SB28 cells have higher self-renewal and tumorigenic capacity. (A) mKate $2^{+}$and $\mathrm{mKate} 2^{-}$cells were cultured at decreasing concentrations with 12 technical replicates. Three biological replicates were performed, and stem cell frequency and the confidence interval are shown. (B) C57BL/6 mice were implanted with equal numbers of mKate $2^{+}$and mKate2- SB28 cells at decreasing concentrations. Kaplan-Meier curves depict the median survival of tumorbearing mice, demonstrating that mice implanted with mKate $2^{+} \mathrm{SB} 28$ cells succumbed to tumor earlier. $\mathrm{p}=0.05$ as determined by log-rank test. 


\section{A}

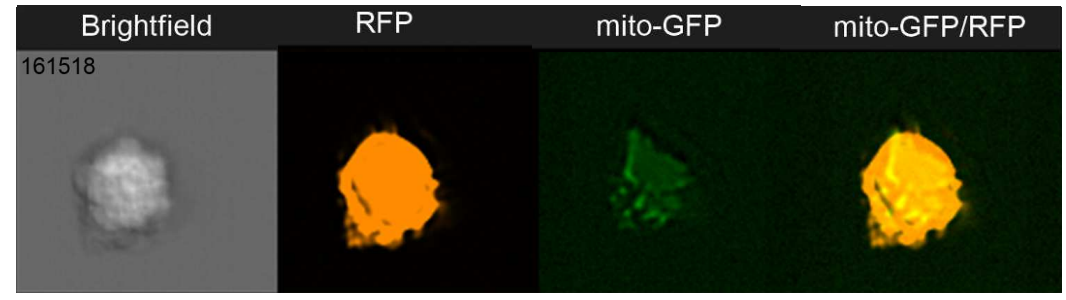

B
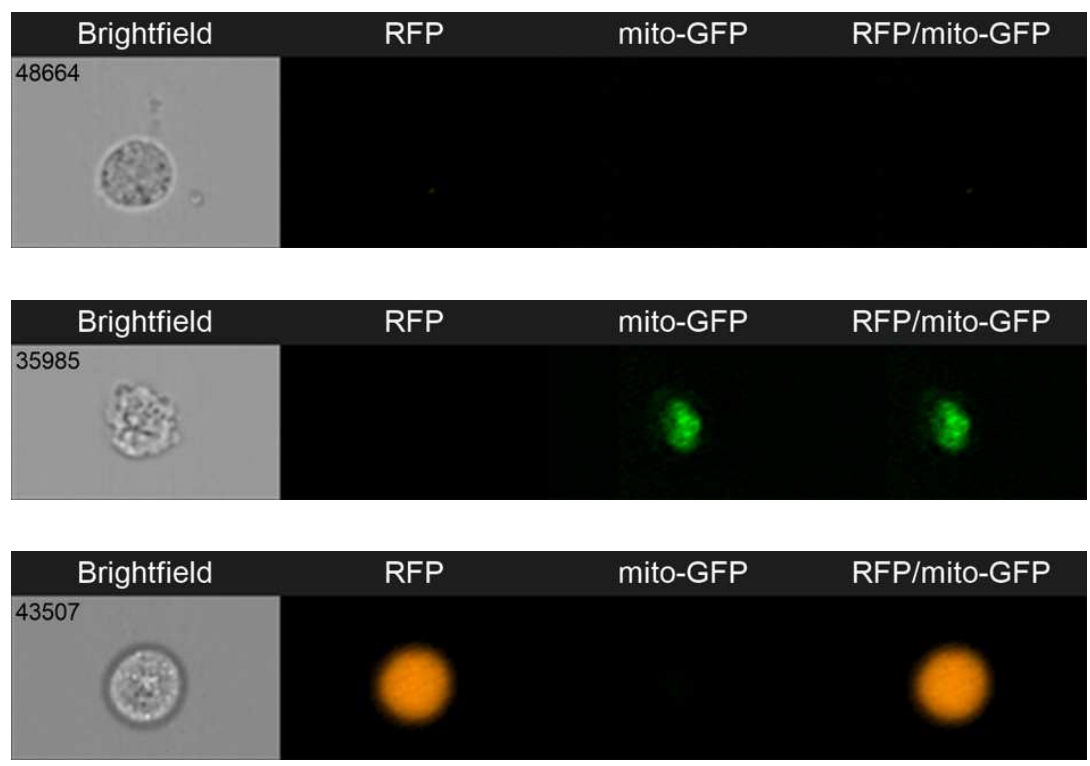

Fig. S11: PDX cells acquire mitochondria from human astrocytes. (A) PDX line JX22 was incubated with GFP-mito-expressing normal human astrocytes for $24 \mathrm{~h}$ at a 1:1 ratio. Mitochondrial puncta in tumor cells were visualized with ImageStream. (B) Single-cell controls demonstrating the specificity of the RFP (tumor) and GFP (mitochondria/astrocyte) signals. 
Gene List

Upregulated Ndufb11; Ddt; Rnaseh2c; Bola3; Sh3bgrl3; 3110039M20Rik; 1810049J17Rik; Polr2i; Lamtor2; Ndufs5; Myl6b; 2010204K13Rik; Gsta4; Higd2a; 2210008F06Rik; Ropn11; Timm13; Gm26792; Rp19-ps6; Pih1d2; Lockd; Lamtor4; Tomm6; Gm9887; G430095P16Rik; Mt2; Snapc5; Snx22; Rpl38-ps2; Pin4; Nrtn; 2010001A14Rik; Immp21; 2310009A05Rik; Epb4114aos; Gm11585; Gm13292; Pcbd2; mt-Tn; Smim4; Pet100; Rpl61; Gm12184; mt-Ta; Gm13436; Serf1; S100a13; Gm8430; Gm2000; Rps26-ps1; Cyp1a1; Gm14586; Gm5905; Gm9843; Tagln; Rps12-ps4; mt-Tc; Gm14303; Gm6204; Mir703; Gm11808; Gm26384

Downregulated Six3os1; Gm21992; Olig2; Rgs8; Zfp874b; Bod11; Golgb1; Dennd6b; Cntrl; Gm45884; Firre; Mki67; Slc22a17; Zfp940; Gm43737; A930033H14Rik; Gm20721; Akna; Cenpe; Akap9; AC160637.1; Tpr; Cep290; Gm16973

Table S1. Differentially expressed genes between $\mathrm{mKate}^{+}$and mKate2- cells that were used for pathway analysis. Genes that were up- or down-regulated $>1.5$-fold with a p-value $<0.05$

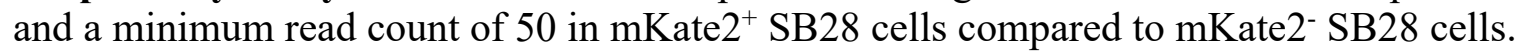


Movie S1. Time-lapse confocal images of in vitro mitochondrial transfer. Astrocytes from mito::mKate2 mice were co-cultured with GFP-expressing SB28 cells for 16 hours before live imaging. Full-thickness z-stacks were obtained at 10-minute intervals. Movie depicts a mito::mKate $2^{+}$astrocyte (magenta) in contact with a GFP-expressing SB28 cell (green). An mKate $2^{+}$mitochondrion is transferred to the dividing GBM cell, which shuttles the mitochondrion back and forth along an intercellular connection linking the GBM daughter cells. At the end of the video, the $\mathrm{mKate} 2^{+}$mitochondrion is retained by the lower daughter cell. See also Fig. 2D for still images with accompanying z-reconstructions. 